\title{
Atmospheres and radiating surfaces of neutron stars with strong magnetic fields
}

\author{
Alexander Y. Potekhin ${ }^{*}$ abcd Wynn C. G. Ho, ${ }^{e}$ Gilles Chabrier $^{d f}$ \\ ${ }^{a}$ Ioffe Institute, Politekhnicheskaya 26, Saint Petersburg, 194021, Russia \\ ${ }^{b}$ St Petersburg Polytechnic University, Politeknicheskaya 29, Saint Petersburg, 195251, Russia \\ ${ }^{c}$ Central Astronomical Observatory at Pulkovo, Pulkovskoe Shosse 65, Saint Petersburg, 196140, \\ Russia \\ ${ }^{d}$ Centre de Recherche Astrophysique de Lyon, Université de Lyon, Université Lyon 1, \\ Observatoire de Lyon, Ecole Normale Supérieure de Lyon, CNRS, UMR 5574, 46 allée d'Italie, \\ 69364, Lyon Cedex 07, France \\ ${ }^{e}$ Mathematical Sciences, Physics \& Astronomy, and STAG Research Centre, University of \\ Southampton, Southampton, SO17 1BJ, UK \\ ${ }^{f}$ School of Physics, University of Exeter, Exeter, EX4 4QL, UK \\ E-mail: palexdastro.ioffe.ru, wynnhodslac.stanford.edu, \\ gilles.chabrierdens-lyon.fr
}

\begin{abstract}
We review the current status of the theory of thermal emission from the surface layers of neutron stars with strong magnetic fields $B \sim 10^{10}-10^{15} \mathrm{G}$, including formation of the spectrum in a partially ionized atmosphere and at a condensed surface. In particular, we describe recent progress in modeling partially ionized atmospheres of central compact objects in supernova remnants, which may have moderately strong fields $B \sim 10^{10}-10^{11} \mathrm{G}$. Special attention is given to polarization of thermal radiation emitted by a neutron star surface. Finally, we briefly describe applications of the theory to observations of thermally emitting isolated neutron stars.
\end{abstract}

The Modern Physics of Compact Stars 2015 30 September 2015 - 3 October 2015

Yerevan, Armenia

${ }^{*}$ Speaker. 


\section{Introduction}

One of the first expectations of neutron-star astrophysics is the possibility to detect thermal radiation from their hot surfaces, whose temperature $T \sim 10^{6} \mathrm{~K}$ implies the maximum of the thermal spectrum in the range of hundreds $\mathrm{eV}[1,2]$. However, the first discovered $\mathrm{X}$-ray sources beyond the Solar system [3-5] appeared to be unrelated with the neutron star radiation [5]. After Sco $\mathrm{X}-1$, the first discovered cosmic X-ray source [3], had also been detected in the optical band [6], Shklovsky [7] argued that its X-ray radiation originated from the accretion of matter onto a neutron star from its companion. This conjecture was initially refuted [8], but later proved to be true [9]. However, the first detection of thermal radiation from the surface of isolated neutron stars (INSs) $[10,11]$ had to wait the launch of focusing X-ray telescopes Einstein (1978-1981) and EXOSAT (1983-1986), which allowed for a dramatic increase in sensitivity to faint point X-ray sources. The next significant advance was due to the ROSAT X-ray observatory (1990-1998), which was the first to reliably identify spectra of the thermal radiation from several pulsars $[12,13]$. In the $21 \mathrm{st}$ century, the data collected by the new generation X-ray observatories Chandra and XMM-Newton give a further impetus to the studies of thermal radiation of neutron stars.

One of the main challenges in the analysis of the neutron star spectra is to disentangle different emission components, overlapping in the X-ray energy range. After discriminating thermal emission, a detailed study of the thermal spectra can yield precious information about the neutron star surface composition and temperature and magnetic field distributions, the properties of dense magnetized plasmas in their envelopes and atmospheres, and eventually constraint the equation of state (EOS) of the ultradense matter in the neutron star cores.

The number of known neutron stars with an unambiguously identified thermal component in the spectrum is not large, but it steadily increases. Some of them can be understood with models of non-magnetic atmospheres, whereas others are believed to be endowed with strong magnetic fields. The non-magnetic neutron-star atmospheres have been studied in many works (see Sect. 2.3 for magnetic model spectra). For the atmospheres composed of hydrogen, databases of model spectra have been published (models NSAGRAV [14], NSSPEC [15], and NSATMOS [16] in the database XSPEC [17]), and a computer code for their calculation has been released [18]. Model spectra were also calculated for different chemical compositions [15, 16, 19-22] and mixtures $[15,20]$. Databases of model spectra have been published for the atmospheres composed of iron and of the solar mixture of elements (NSSPEC [15]), of helium (NSX [21]), and of carbon (NSX [21] and CARBATM [22]). Examples of thermal spectra successfully interpreted with the non-magnetic atmosphere models include bursters, soft X-ray transients in quiescence, some central compact objects (CCOs) in supernova remnants, and some millisecond pulsars (see the table in the review [23]). Measuring the basic parameters of neutron stars using model non-magnetic atmospheres has been also recently reviewed in [24].

Here, however, we will focus exclusively on models of those thermal spectra of the INSs that are significantly affected by strong magnetic fields (see [23] for more detailed discussion). The paper is organized as follows. In Sect. 2 we overview the theory of partially ionized neutron star atmospheres with strong magnetic fields. In Sect. 3 we describe the model of a condensed radiating surface and hybrid models of a condensed surface covered by a thin atmosphere. Section 4 is devoted to the polarization of the neutron star thermal radiation that may be measured in fu- 
ture. Available examples of successful applications of the theory to observations are overviewed in Sect. 5, and conclusions are given in Sect. 6.

\section{Theory of strongly magnetized neutron-star atmospheres}

We call an atmosphere strongly magnetized, if the magnetic field $B$ strongly (non-perturbatively) affects its opacities and radiative transfer for thermal photons. This is the case if the electron cyclotron energy $\hbar \omega_{\mathrm{c}} \approx 11.577 B_{12} \mathrm{keV}$ is greater than either the photon energies $\hbar \omega$ or the atomic binding energies, or both. Here, $\omega$ is the photon angular frequency, $\omega_{\mathrm{c}}=e B / m_{\mathrm{e}} c$ is the electron cyclotron frequency, $m_{\mathrm{e}}$ and $-e$ are the electron mass and charge, $c$ is the speed of light, and $B_{12} \equiv B /\left(10^{12} \mathrm{G}\right)$. These two conditions imply

$$
B \gtrsim \frac{m_{\mathrm{e}} c}{\hbar e} k_{\mathrm{B}} T \sim 10^{10} T_{6} \mathrm{G}, \quad B \gtrsim B_{0}=\frac{m_{\mathrm{e}}^{2} c e^{3}}{\hbar^{3}}=2.3505 \times 10^{9} \mathrm{G},
$$

where $T_{6}=T / 10^{6} \mathrm{~K}$. The quantity $B_{0}$ in Eq. (2.1) determines the atomic magnetic-field parameter $\gamma \equiv B / B_{0}$. It is also convenient to define the relativistic magnetic-field parameter $b \equiv \hbar \omega_{\mathrm{c}} / m_{\mathrm{e}} c^{2}=$ $B_{12} / 44.14$. We call magnetic field superstrong if $b \gtrsim 1$. In this case the specific effects of Quantum Electrodynamics (QED) become quite important. The superstrong fields are believed to exist at the surfaces of magnetars and high- $B$ pulsars (see the reviews [25-27]).

\subsection{Radiative transfer in normal modes}

As has been demonstrated in [28], at typical conditions in neutron star photospheres one can describe radiative transfer in terms of specific intensities of two normal polarization modes [29]. This is true if the magnetic field is strong and $\omega$ lies outside narrow frequency ranges near resonances and above the electron plasma frequency $\omega_{\mathrm{pe}}=\left(4 \pi e^{2} n_{\mathrm{e}} / m_{\mathrm{e}}\right)^{1 / 2}$, where $n_{\mathrm{e}}$ is the electron number density. The normal modes, called extraordinary (X-mode, denoted by subscript or superscript $j=1$ or $\mathrm{X}$ ) and ordinary (O-mode, $j=2$ or O), have different polarization vectors $\boldsymbol{e}_{j}$ and different absorption and scattering coefficients, which depend on the angle $\theta_{k B}$ between the wave vector $\boldsymbol{k}$ and the magnetic field $\boldsymbol{B}$. The radiative transfer equations for the two normal modes read [30]

$$
\cos \theta_{k} \frac{\mathrm{d} I_{\omega, j}(\hat{\mathbf{k}})}{\mathrm{d} y_{\mathrm{col}}}=\varkappa_{\omega, j}(\hat{\mathbf{k}}) I_{\omega, j}(\hat{\mathbf{k}})-\frac{1}{2} \varkappa_{\omega, j}^{\mathrm{a}}(\hat{\mathbf{k}}) \mathscr{B}_{\omega, T}-\sum_{j^{\prime}=1}^{2} \int_{(4 \pi)} \varkappa_{\omega, j^{\prime} j}^{\mathrm{s}}\left(\hat{\mathbf{k}}^{\prime}, \hat{\mathbf{k}}\right) I_{\omega, j^{\prime}}\left(\hat{\mathbf{k}}^{\prime}\right) \mathrm{d} \Omega_{\boldsymbol{k}^{\prime}},
$$

where $I_{\omega, j}$ denotes the specific intensity of the polarization mode $j$ per unit circular frequency (if $I_{V}$ is the specific intensity per unit frequency, then $\left.I_{\omega}=I_{V} /(2 \pi)[31]\right), \hat{\mathbf{k}}$ is the unit vector along the wave vector $\boldsymbol{k}$,

$$
\mathscr{B}_{\omega, T}=\frac{\hbar \omega^{3}}{4 \pi^{3} c^{2}}\left(\mathrm{e}^{\hbar \omega / k_{\mathrm{B}} T}-1\right)^{-1}
$$

is the specific intensity of non-polarized blackbody radiation,

$$
\varkappa_{\omega, j}(\hat{\mathbf{k}}) \equiv \varkappa_{\omega, j}^{\mathrm{a}}(\hat{\mathbf{k}})+\sum_{j^{\prime}=1}^{2} \int_{(4 \pi)} \varkappa_{\omega, j^{\prime} j}^{\mathrm{s}}\left(\hat{\mathbf{k}}^{\prime}, \hat{\mathbf{k}}\right) \mathrm{d} \Omega_{\boldsymbol{k}^{\prime}}
$$


is the total opacity, $\varkappa_{\omega, j}^{\mathrm{a}}$ and $\varkappa_{\omega, j^{\prime} j}^{\mathrm{s}}\left(\hat{\mathbf{k}}^{\prime}, \hat{\mathbf{k}}\right)$ are its components due to, respectively, the true absorption and the scattering that changes the ray direction from $\hat{\mathbf{k}}^{\prime}$ to $\hat{\mathbf{k}}$ and may also change the polarization from $j^{\prime}$ to $j, \mathrm{~d} \Omega_{k} \equiv \sin \theta_{k} \mathrm{~d} \theta_{k} \mathrm{~d} \varphi_{k}$ is a solid angle element, $\theta_{k}$ is the angle between $k$ and the normal to the surface $\mathbf{n}$, and $\varphi_{k}$ is the angle in the surface plane. Finally, $y_{\mathrm{col}}=\int_{r}^{\infty}\left(1+z_{g}\right) \rho(r) \mathrm{d} r$ is the column density, where the factor $1+z_{g}=\left(1-x_{g}\right)^{-1 / 2}$ is the scale change due to the gravity according to the General Relativity (GR), $z_{g}$ is the gravitational redshift, $x_{g}=2 G M / c^{2} R \approx$ $\left(M / M_{\odot}\right)(2.95 \mathrm{~km} / R)$ is the compactness parameter of the star with mass $M$ and radius $R$, and $G$ is the gravitational constant (for a typical neutron star $x_{g}$ lies between $1 / 5$ and $1 / 2$ ). The radiative transfer equations should be supplemented with the hydrostatic and energy balance equations (see [32]).

The major axis of the polarization ellipse is transverse to $\boldsymbol{B}$ for the $\mathrm{X}$-mode and coplanar with $\boldsymbol{B}$ for the O-mode. Since the magnetic field hampers electron motion in the transverse direction, it also suppresses the damping of the $\mathrm{X}$-wave by the electrons, making the opacities of the $\mathrm{X}$-mode strongly reduced, if $\omega_{\mathrm{c}} \gg \omega$. The dependence of the opacities on $\hat{\mathbf{k}}$ and $\hat{\mathbf{k}}^{\prime}$ is also affected by $\boldsymbol{B}$. Therefore, the emission of a magnetized atmosphere depends on the angles $\theta_{k}, \varphi_{k}$, and the angle $\theta_{n B}$ between $\boldsymbol{B}$ and $\mathbf{n}$, unlike in the non-magnetic case where it depends only on $\theta_{k}$. In practice, one usually neglects the dependence of scattering on the angle between $\hat{\mathbf{k}}$ and $\hat{\mathbf{k}}^{\prime}$, in which case (called the approximation of isotropic scattering $) \varkappa_{\omega, j^{\prime} j}^{\mathrm{s}}\left(\hat{\mathbf{k}}^{\prime}, \hat{\mathbf{k}}\right)$ depends only on the angles $\theta_{k B}$ and $\theta_{k B}^{\prime}$ that the wave vectors $\boldsymbol{k}$ and $\boldsymbol{k}^{\prime}$ make with $\boldsymbol{B}$.

The polarization vectors of normal modes $\boldsymbol{e}_{\omega, j}$ are determined by the complex dielectric tensor $\boldsymbol{\varepsilon}(\omega)$ and magnetic permeability tensor. The latter can be set equal to the unit tensor $\mathbf{I}$ for a gaseous plasma (see [29]). In the Cartesian system with unit vectors $\hat{\mathbf{e}}_{x, y, z}$ such that $\hat{\mathbf{e}}_{z}$ is directed along $\boldsymbol{B}$,

$$
\boldsymbol{\varepsilon}=\mathbf{I}+4 \pi \chi=\left(\begin{array}{ccc}
\varepsilon_{\perp} & \mathrm{i} \varepsilon_{\wedge} & 0 \\
-\mathrm{i} \varepsilon_{\wedge} & \varepsilon_{\perp} & 0 \\
0 & 0 & \varepsilon_{\|}
\end{array}\right)
$$

where $\boldsymbol{\chi}=\boldsymbol{\chi}^{\mathrm{H}}+\mathrm{i} \boldsymbol{\chi}^{\mathrm{A}}$ is the complex polarizability tensor of plasma, $\boldsymbol{\chi}^{\mathrm{H}}$ and $\boldsymbol{\chi}^{\mathrm{A}}$ are its Hermitian and anti-Hermitian parts, respectively. The anti-Hermitian part $\chi^{\mathrm{A}}(\omega)$ is determined by the absorption opacities, and the Hermitian part $\chi^{\mathrm{H}}(\omega)$ can be obtained from $\chi^{\mathrm{A}}(\omega)$ using the KramersKronig relation $[33,34]$.

In strong magnetic fields, specific QED effects called polarization and magnetization of vacuum can be important (see, e.g., [35]). At $B \lesssim 10^{16}$, the vacuum polarization is weak. Then the total dielectric tensor is $\boldsymbol{\varepsilon}=\mathbf{I}+4 \pi \boldsymbol{\chi}(\omega)+4 \pi \boldsymbol{\chi}^{\mathrm{vac}}$, where the vacuum polarizability tensor $\boldsymbol{\chi}^{\mathrm{vac}}$ does not depend on $\omega$. In the Cartesian coordinate system with $\hat{\mathbf{e}}_{z}$ along $\boldsymbol{B}$, the tensors of vacuum polarizability $\boldsymbol{\chi}^{\mathrm{vac}}$ and permeability become diagonal, with the coefficients determined by only three numbers, called vacuum polarizability and magnetization coefficients. These coefficients were obtained in [36] at $b \ll 1$ and in [37] at $b \gg 1$. At arbitrary $b$, they were calculated in [38] and fitted by simple analytic functions in [34]. Convenient expressions of the components of $\boldsymbol{e}_{j}$ through the components of $\boldsymbol{\chi}(\omega)$ and the vacuum polarization and magnetization coefficients have been presented in [39].

In the approximation of isotropic scattering, at a given frequency $\omega$, the opacities can be 
written in the form (e.g., [30])

$$
\varkappa_{j}^{\mathrm{a}}=\sum_{\alpha=-1}^{1}\left|e_{j, \alpha}\left(\theta_{k B}\right)\right|^{2} \frac{\sigma_{\alpha}^{\mathrm{a}}}{m_{\mathrm{i}}}, \quad \varkappa_{j j^{\prime}}^{\mathrm{s}}=\frac{3}{4} \sum_{\alpha=-1}^{1}\left|e_{j, \alpha}\left(\theta_{k B}\right)\right|^{2} \frac{\sigma_{\alpha}^{\mathrm{s}}}{m_{\mathrm{i}}} \int_{0}^{\pi}\left|e_{j^{\prime}, \alpha}\left(\theta_{k B}^{\prime}\right)\right|^{2} \sin \theta_{k B}^{\prime} \mathrm{d} \theta_{k B}^{\prime},
$$

where $\alpha=0, \pm 1$, and $\sigma_{\alpha}^{\mathrm{a}, \mathrm{s}}$ are the absorption and scattering cross-sections for the three basic cyclic polarizations with $\boldsymbol{e}_{0}=\boldsymbol{e}_{z}$ and $\boldsymbol{e}_{ \pm 1}=\left(\boldsymbol{e}_{x} \pm \mathrm{i} \boldsymbol{e}_{y}\right) / \sqrt{2}$. The absorption cross-sections include contributions of photon interaction with free electrons or ions in the plasma environment (free-free transitions) as well as with bound states of atoms and ions (bound-bound and bound-free transitions).

At large optical depth $\tau_{\omega}=\int_{r}^{\infty} \varkappa_{\omega}^{\text {eff }}\left(r^{\prime}\right) \mathrm{d} y_{\text {col }}\left(r^{\prime}\right)$, where $\varkappa_{\omega}^{\text {eff }}$ is the effective opacity defined below, radiation is almost isotropic: the magnitude of the diffusive radiative flux $F_{\omega, j}$ is much smaller than the mean intensity $J_{\omega, j}$, where

$$
J_{\omega, j}=\frac{1}{4 \pi} \int_{(4 \pi)} I_{\omega, j}(\hat{\mathbf{k}}) \mathrm{d} \Omega_{k}, \quad \boldsymbol{F}_{\omega, j}=\int_{(4 \pi)} I_{\omega, j}(\hat{\mathbf{k}}) \hat{\mathbf{k}} \mathrm{d} \Omega_{k} .
$$

In this case an approximate solution to (2.2) is provided by the diffusion approximation [30]:

$$
I_{\omega, j}(\hat{\mathbf{k}}) \approx J_{\omega, j}+\frac{3}{4 \pi} \boldsymbol{F}_{\omega, j} \cdot \hat{\mathbf{k}}, \quad \frac{\mathrm{d}}{\mathrm{d} y_{\mathrm{col}}} D_{\omega, j} \frac{\mathrm{d}}{\mathrm{d} y_{\mathrm{col}}} J_{\omega, j}=\bar{\varkappa}_{\omega, j}^{\mathrm{a}}\left[J_{\omega, j}-\frac{\mathscr{B} \omega, T}{2}\right]+\bar{\varkappa}_{\omega}^{\mathrm{s}}\left[J_{\omega, j}-J_{\omega, 3-j}\right] .
$$

Here, the average absorption and scattering opacities are

$$
\bar{\varkappa}_{\omega, j}^{\mathrm{a}}=\frac{1}{4 \pi} \int_{(4 \pi)} \varkappa_{\omega, 12}^{\mathrm{a}} \mathrm{d} \Omega_{k}, \quad \bar{\varkappa}_{\omega}^{\mathrm{s}}=\frac{1}{4 \pi} \int_{(4 \pi)} \mathrm{d} \Omega_{k^{\prime}} \int_{(4 \pi)} \mathrm{d} \Omega_{k} \varkappa_{\omega, 12}^{\mathrm{s}}\left(\hat{\mathbf{k}}^{\prime}, \hat{\mathbf{k}}\right),
$$

and the diffusion coefficient is

$$
D_{\omega, j}=\frac{\cos ^{2} \theta_{n B}}{3 \varkappa_{\omega, j}^{\|}}+\frac{\sin ^{2} \theta_{n B}}{3 \varkappa_{\omega, j}^{\perp}} \text {, where }\left\{\begin{array}{c}
\left(\varkappa_{\omega, j}^{\|}\right)^{-1} \\
\left(\varkappa_{\omega, j}^{\perp}\right)^{-1}
\end{array}\right\}=\frac{3}{4} \int_{0}^{\pi}\left\{\begin{array}{c}
2 \cos ^{2} \theta_{k B} \\
\sin ^{2} \theta_{k B}
\end{array}\right\} \frac{\sin \theta_{k B} \mathrm{~d} \theta_{k B}}{\varkappa_{\omega, j}\left(\theta_{k B}\right)} .
$$

The effective opacity for non-polarized radiation is $\varkappa_{\omega}^{\text {eff }}=2 /\left(3 D_{\omega, 1}+3 D_{\omega, 2}\right)$. The diffusion approximation (2.8) serves as a starting point to an iterative method [40], which allows one to solve the system (2.2) more accurately.

To solve the radiative transfer equations, one must know the dependencies of the temperature and densities of atoms, ions, and electrons on the depth. These dependencies can be found from the equations of thermal, hydrostatic, and ionization equilibrium supplemented with the EOS. In the plane-parallel limit, the condition of radiative thermal equilibrium requires the outward flux

$$
F_{\mathrm{ph}}=\int_{0}^{\infty} \mathrm{d} \omega \int_{(4 \pi)} \sum_{j=1}^{2} I_{\omega, j}(\hat{\mathbf{k}}) \cos \theta_{k} \mathrm{~d} \Omega_{k}
$$

to remain constant throughout the atmosphere. In the approximation of isotropic scattering, the equations of hydrostatic balance are (cf. [32])

$$
\frac{\mathrm{d} P}{\mathrm{~d} y_{\mathrm{col}}}=g-g_{\mathrm{rad}}, \quad g_{\mathrm{rad}} \approx \frac{1}{c} \int_{0}^{\infty} \mathrm{d} \omega \int \cos \theta_{k} \sum_{j=1}^{2} \varkappa_{\omega, j}(\hat{\mathbf{k}}) I_{\omega, j}(\hat{\mathbf{k}}) \mathrm{d} \Omega_{k} .
$$




\subsection{Physics input}

To solve the radiation transfer problem formulated in Sect. 2.1, we need to know the composition of the plasma, its EOS, and absorption and scattering cross-sections. All these ingredients are affected by a strong magnetic field. There have been many works devoted to these effects. We will give only a brief sketch of the basic results (see [23] for details).

The motion of charged particles in a magnetic field $\boldsymbol{B}$ is quantized in discrete Landau levels, whereas the longitudinal (parallel to $\boldsymbol{B}$ ) momentum of the particle is continuous. The threshold excitation energy of the $N$ th Landau level equals $E_{N}=m_{\mathrm{e}} c^{2}(\sqrt{1+2 b N}-1)$. The wave functions that describe an electron in a magnetic field [41] have a characteristic transverse scale of the order of the "magnetic length" $a_{\mathrm{m}}=(\hbar c / e B)^{1 / 2}=a_{\mathrm{B}} / \sqrt{\gamma}$, where $a_{\mathrm{B}}=\hbar^{2} / m_{\mathrm{e}} e^{2}$ is the Bohr radius.

In practice, Landau quantization becomes important when $N$ is not too large. For the electrons in a plasma at equilibrium this means that the electron cyclotron energy $\hbar \omega_{\mathrm{c}}$ is at least comparable to both the electron Fermi energy $\varepsilon_{\mathrm{F}}$ and temperature $k_{\mathrm{B}} T$ in energy units. If $\hbar \omega_{\mathrm{c}}$ is appreciably larger than both these energies, then most electrons reside on the ground Landau level, and the field is called strongly quantizing. For a plasma of electrons and ions with charge $Z e$ and mass $m_{\mathrm{i}}=A m_{\mathrm{u}}$, where $m_{\mathrm{u}}=1.66054 \times 10^{-24} \mathrm{~g}$, the field is strongly quantizing if both conditions $\rho<\rho_{B}$ and $\zeta_{\mathrm{e}} \gg 1$ are satisfied, where

$$
\rho_{B}=\frac{m_{\mathrm{i}}}{\pi^{2} \sqrt{2} a_{\mathrm{m}}^{3} Z}=7045 \frac{A}{Z} B_{12}^{3 / 2} \mathrm{~g} \mathrm{~cm}^{-3}, \quad \zeta_{\mathrm{e}}=\frac{\hbar \omega_{\mathrm{c}}}{k_{\mathrm{B}} T}=134.34 \frac{B_{12}}{T_{6}} .
$$

In neutron star atmospheres, as a rule, the fields $B \gg 10^{10} \mathrm{G}$ are strongly quantizing. In the opposite case, where electrons are smoothly distributed over a large number of the Landau levels, the field can be considered as non-quantizing. In the magnetospheres, which have lower densities, electrons can condensate on the lowest Landau level even at $B \sim 10^{8} \mathrm{G}$ because of the violation of the LTE conditions (e.g., [42]), but this is not the case in the photospheres (see [23]).

For ions, the parameter $\zeta_{\mathrm{e}}$ is replaced by $\zeta_{\mathrm{i}}=\hbar \omega_{\mathrm{ci}} / k_{\mathrm{B}} T=0.0737(Z / A) B_{12} / T_{6}$, where $\omega_{\mathrm{ci}}=$ $Z e B /\left(m_{\mathrm{i}} c\right)$ is the ion cyclotron frequency. In magnetar atmospheres, the parameter $\zeta_{\mathrm{i}}$ is not small, and the quantization of the ion motion should be taken into account. A parameter analogous to $\rho_{B}$ is unimportant for ions, because they are non-degenerate in neutron star atmosphere.

Scattering cross-sections in neutron star atmospheres are well known [30, 42, 43]. The scattering on electrons is strongly reduced compared to the zero-field case at $\omega \ll \omega_{\mathrm{c}}$ and exhibits a cyclotron resonance at $\omega_{\mathrm{c}}$. The ion scattering cross-section is analogous. In superstrong fields one cannot neglect the scattering on ions, because of the resonance at $\omega_{\mathrm{ci}}$. In addition, in a quantizing magnetic field, a photon can be absorbed or emitted by a free electron in a transition between Landau levels. In the non-relativistic or dipole approximation, such transitions occur between the neighboring levels at the fundamental cyclotron frequency $\omega_{\mathrm{c}}$. In the relativistic theory, the multipole expansion gives rise to cyclotron harmonics [31]. They manifest themselves in the opacities when one goes beyond the cold plasma approximation. Absorption cross-sections at these harmonics were derived in [44] and represented in a convenient form in [45].

The quantization of electron motion leads to the appearance of cyclotron harmonics in the freefree absorption as well, even in the non-relativistic theory. Photon absorption cross-sections for an electron, which moves in a quantizing magnetic field and interacts with a non-moving point charge, were derived in [46]. However, an ion can be considered as non-moving only if $\omega \gg \omega_{\mathrm{ci}}$ [29]. In 
the superstrong field of magnetars, the latter condition is unacceptable. A more accurate treatment of absorption of a photon by the system of a finite-mass ion and an electron was performed in $[47,48]$. According to the latter studies, the free-free absorption cross-section $\sigma_{\alpha}^{\mathrm{a}(\mathrm{ff})}(\omega)$ has peaks at the multiples of both the electron and ion cyclotron frequencies for all polarizations $\alpha$. However, these two types of peaks are different. Unlike the electron cyclotron harmonics, the ion cyclotron harmonics are so weak that they can be safely neglected in the neutron star atmospheres.

As first noticed in [49], atoms with bound states should be much more abundant at $B \gg B_{0}$ than at $B \lesssim B_{0}$ in a neutron star atmosphere at the same temperature. This difference is caused by the increase of atomic binding energies and decrease of atomic sizes at $B \gg B_{0}$. Therefore it is important to consider the bound states and bound-bound and bound-free transitions in a strong magnetic field even for light-element atmospheres, which would be almost fully ionized in the non-magnetic case.

Many authors studied atoms with an infinitely heavy (fixed in space) nucleus in strong magnetic fields (see, e.g., [50, 51], for review). This model, however, can be considered only as a first approximation. If $\gamma=B / B_{0}$ is not negligibly small compared to the nucleus-to-electron mass ratio $m_{\mathrm{i}} / m_{\mathrm{e}}$, one should take into account quantum oscillations of an atomic nucleus, which are different for different atomic quantum states. Moreover, the astrophysical simulations assume finite temperatures, hence thermal motion of particles. The theory of motion of a system of point charges $q_{i}$ in a constant magnetic field was reviewed in [52]. Instead of the canonical momentum $\boldsymbol{P}$, a relevant conserved quantity is pseudomomentum $\boldsymbol{K}=\boldsymbol{P}+(1 / 2 c) \boldsymbol{B} \times \sum_{i} q_{i} \boldsymbol{r}_{\boldsymbol{i}}$. For a hydrogen atom, $\boldsymbol{K}=\boldsymbol{P}-(e / 2 c) \boldsymbol{B} \times \boldsymbol{r}$, where $\boldsymbol{r}$ connects the proton and the electron [53]. The specific effects related to collective motion of a system of charged particles are especially important in neutron star atmospheres at $B \gg B_{0}$. In particular, so called decentered states may become populated, where an electron is localized mostly in a "magnetic well" aside from the Coulomb center. Numerical calculations of the energy spectrum of the hydrogen atom with allowance for the effects of motion across a strong magnetic field were performed in [54, 55]. Probabilities of various radiative transitions for a hydrogen atom moving in a strong magnetic field were studied in [55-57].

Quantum-mechanical calculations of the characteristics of a $\mathrm{He}^{+}$ion moving across a strong magnetic field are performed in $[58,59]$. The basic difference from the case of a neutral atom is that the the ion motion is restricted by the field in the transverse plane, therefore the values of $K^{2}$ are quantized (see [52]).

Currently there is no detailed calculation of binding energies, oscillator strengths, and photoionization cross-sections for atoms and ions other than $\mathrm{H}_{\text {and }} \mathrm{He}^{+}$, arbitrarily moving in a strong magnetic field. For such species one usually neglects the decentered states and uses a perturbation theory with respect to $K_{\perp}[60,61]$. An order-of-magnitude estimate [23] of the validity of the latter gives, for an atom with mass $m_{\mathrm{a}}=A m_{\mathrm{u}}$, the condition $k_{\mathrm{B}} T / E_{\mathrm{b}} \ll m_{\mathrm{a}} /\left(\gamma m_{\mathrm{e}}\right) \approx 4 A / B_{12}$, where $E_{\mathrm{b}}$ is the atomic ionization energy. It is satisfied for low-lying levels of carbon and heavier ions, if $B \lesssim 10^{13} \mathrm{G}$ and $T \lesssim 10^{6} \mathrm{~K}$ (see the discussion in [23]). A practical method of calculations of the quantum-mechanical characteristics of multielectron atoms and ions, based on a combination of several perturbation theories with respect to different physical parameters, has been developed in [62].

Since the quantum-mechanical characteristics of an atom in a strong magnetic field depend on $K_{\perp}$, the atomic distribution over $K_{\perp}$ cannot be written in a closed form, and only the distribution 
over longitudinal momenta $K_{z}$ remains Maxwellian. The first EOS calculations with the complete account of these effects have been performed in [63] for hydrogen atmospheres. The same approach with slight modifications was then applied to strongly magnetized helium plasmas [64].

The calculation of the cross-section of photoabsorption by bound states of atoms and ions in the strong magnetic fields implies averaging of the cross-sections over all values of $K_{\perp}$ at every $\omega$. Since the distribution over $K_{\perp}$ is continuous for the atoms and discrete for the ions, such averaging implies an integration over $K_{\perp}$ for atoms and a summation for ions. Statistical weights for this averaging should be consistent with the statistical model of the plasma used in the EOS calculations. To date, such fully self-consistent calculations, including both centered and decentered bound states (i.e., small and large $K_{\perp}$ ) have been realized only for neutron-star atmospheres composed of hydrogen $[47,65,66]$. For atoms and ions with several bound electrons $(\mathrm{C}, \mathrm{O}, \mathrm{Ne})$, calculations have been performed $[67,68]$ in terms of the above-mentioned perturbation theory.

\subsection{Modeling observed spectra}

The strong gravity of a neutron star induces a significant redshift of the local photon frequency $\omega$ to $\omega_{\infty}=\omega /\left(1+z_{g}\right)$ in the remote observer's reference frame. Accordingly, a thermal spectrum with effective temperature $T_{\text {eff }}$ transforms for the remote observer into a spectrum with a lower "observed" temperature $T_{\text {eff }}^{\infty}=T_{\text {eff }} /\left(1+z_{g}\right)$. Along with the radius $R$ that is determined by the equatorial length $2 \pi R$ in the local reference frame, one often considers an apparent radius for a remote observer, $R_{\infty}=R\left(1+z_{g}\right)$, so that the apparent photon luminosity $L_{\mathrm{ph}}^{\infty}$ is determined by the Stefan-Boltzmann law $L_{\mathrm{ph}}^{\infty}=L_{\mathrm{ph}} /\left(1+z_{\mathrm{g}}\right)^{2}=4 \pi \sigma_{\mathrm{SB}} R_{\infty}^{2}\left(T_{\mathrm{eff}}^{\infty}\right)^{4}$, where $\sigma_{\mathrm{SB}}$ is the Stefan-Boltzmann constant, and $L_{\mathrm{ph}}=4 \pi \sigma_{\mathrm{SB}} R^{2} T_{\text {eff }}^{4}$ is the luminosity in the local reference frame.

Since thermal diffusion is anisotropic in a strong magnetic field, the temperature is nonuniform over the stellar surface, which can lead to modulation of the observed thermal flux as the star rotates [69]. Then it is convenient to define a local effective surface temperature $T_{\mathrm{S}}$ by the relation $F_{\mathrm{ph}}(\theta, \varphi)=\sigma_{\mathrm{SB}} T_{\mathrm{S}}^{4}$, where $F_{\mathrm{ph}}$ is the local radial flux density at the surface point, Eq. (2.11), determined by the polar angle $(\theta)$ and azimuth $(\varphi)$ in the spherical coordinate system. Since $L_{\mathrm{ph}}$ is the integral of $F_{\mathrm{ph}}(\theta, \varphi)$ over the surface, $T_{\mathrm{eff}}^{4}$ is equal to the average of $T_{\mathrm{s}}^{4}$.

Because of the light bending in strong gravity, the observer receives a photon whose wave vector makes a different angle $\theta$ with normal to the surface than the angle $\theta_{k}$ at the point where this photon was emitted. For the compactness parameters $x_{g}<1 / 2$, this effect is well described by the simple approximation [70] $\cos \theta_{k}=x_{g}+\left(1-x_{g}\right) \cos \theta$. The spectral flux that comes to an observer can be most easily calculated using the equations presented in [71] provided that the distribution of $I_{\omega, j}(\hat{\mathbf{k}})$ is known for the entire visible surface of the neutron star. The problem is complicated because of non-uniform surface distributions of the magnetic field and effective temperature. A fiducial model for the magnetic field distribution is the relativistic dipole [72], but recent numerical simulations of the magnetothermal evolution produce more complicated distributions (see [73, 74] and references therein). The temperature distribution, consistent with the magnetic-field distribution, is found from calculations of heat transport in neutron star envelopes (see [75] for review).

Local spectra from strongly magnetized, fully ionized neutron-star atmospheres were calculated in [76, 77]. Later the authors included spectral tables for $B=10^{12}$ and $10^{13} \mathrm{G}$ in the XSPEC package [17] under the name NSA. The authors have shown that the spectra of magnetic hydrogen and helium atmospheres are softer than the respective non-magnetic spectra, but harder than the 

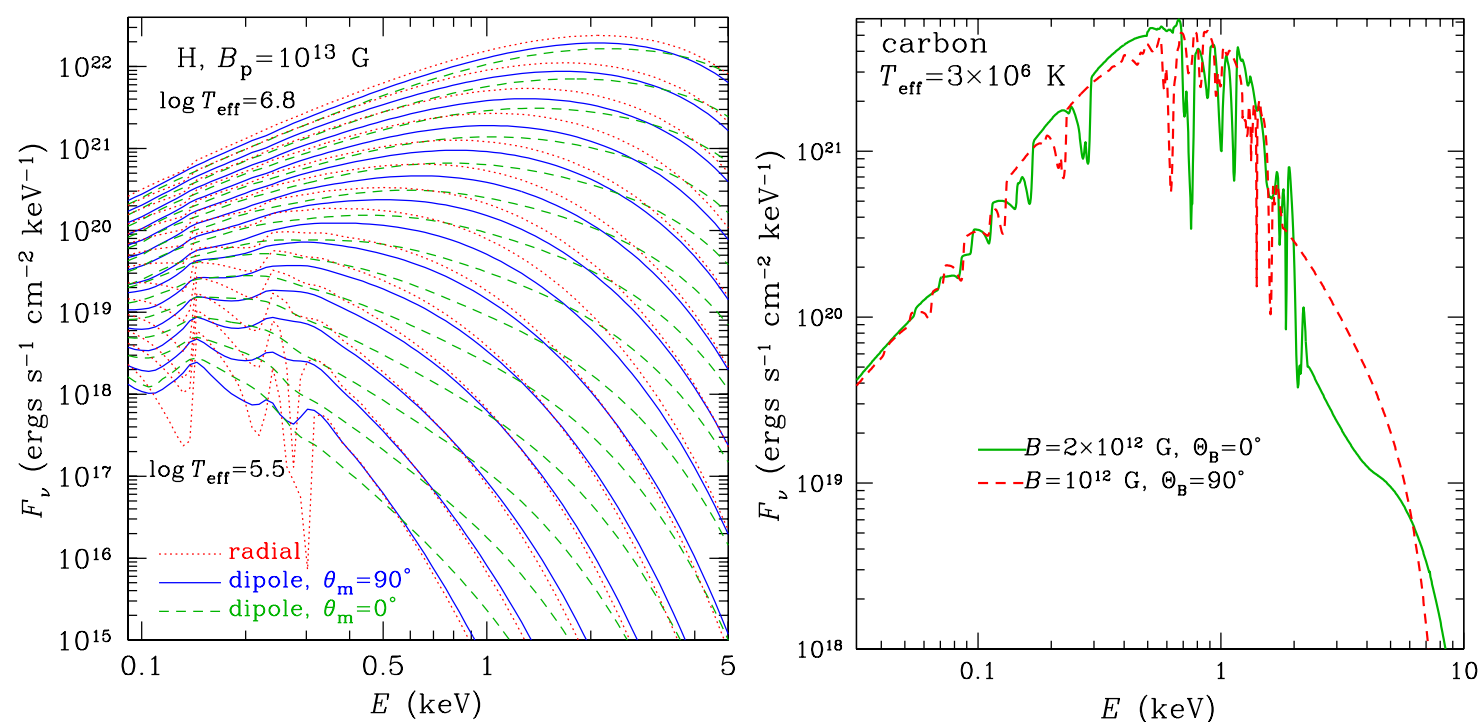

Figure 1: Left panel. Integral spectra of a hydrogen atmosphere of a neutron star with $M=1.4 M_{\odot}, R=12$ $\mathrm{km}$, and with different effective temperatures $T_{\text {eff }}\left(\log T_{\text {eff }}(\mathrm{K})\right.$ from 5.5 to 6.8 with step 0.1$)$. The dashed and solid lines represent the model with a dipole field of strength $B_{\mathrm{p}}=10^{13} \mathrm{G}$ at the pole and oriented along and across the line of sight, respectively. For comparison, the dotted curves show the model with a constant field $B=10^{13} \mathrm{G}$, normal to the surface. Right panel (from [68]). Local spectra at the magnetic pole (solid curve) and equator (dashed curve) for a neutron star with carbon atmosphere, the dipole field with polar strength of $B_{\mathrm{p}}=2 \times 10^{12} \mathrm{G}$ (neglecting the relativistic corrections) and uniform effective temperature $3 \times 10^{6} \mathrm{~K}$.

blackbody spectrum with the same temperature. At contrast to the isotropic blackbody radiation, radiation of a magnetic atmosphere consists of a narrow $\left(<5^{\circ}\right)$ pencil beam along the magnetic field and a broad fan beam with typical angles $\sim 20^{\circ}-60^{\circ}$ (as had been already predicted in [78]).

Later, similar calculations were performed by other research groups $[39,79,80]$, paying special attention to possible manifestations of the ion cyclotron resonance in magnetar spectra. In particular, it was shown $[39,80,81]$ that vacuum polarization leads to conversion of the normal modes: a photon related to one mode transforms, with a certain probability, into a photon of the other mode while crossing a surface at a critical density which depends on $\omega$. For $B \sim 10^{14} \mathrm{G}$ and $\omega \sim \omega_{\mathrm{ci}}$, conversion occurs at a density where the atmosphere is optically thin for the extraordinary mode but optically thick for the ordinary mode. As a result, ordinary photons converting into extraordinary ones will reduce the strength of the ion cyclotron absorption feature in the emission spectrum of magnetars.

A strongly magnetized hydrogen atmosphere model with full account of the partial ionization and the atomic motion effects was constructed in [34]. The calculated spectra revealed a narrow absorption line at the proton cyclotron energy and some features related to atomic transitions. Similar to fully ionized plasma models, the intensity has a maximum at higher energies relative to the maximum of the Planck function, but at lower energies relative to the non-magnetic hydrogen atmosphere model. Therefore, the model of a fully-ionized atmosphere with a strong magnetic field can yield a relatively reliable temperature, although it does not reproduce the spectral features caused by atomic transitions. 
Synthetic spectra of partially ionized hydrogen atmospheres with averaging over the stellar surface with realistic temperature distribution were calculated in [82]. The left panel of Fig. 1 shows examples of such spectra calculated for a neutron star with $M=1.4 M_{\odot}$ and $R=12 \mathrm{~km}$, endowed with a dipole magnetic field, whose value at the magnetic pole is $B_{\mathrm{p}}=10^{13} \mathrm{G}$. For comparison, the dotted lines show spectra calculated for constant $B=10^{13} \mathrm{G}$ for the same values of $T_{\text {eff. }}$. In the dipole configuration, the spectral features are strongly smeared by the averaging over the surface temperature and magnetic field distributions, and the spectrum depends on the magnetic axis orientation $\theta_{\mathrm{m}}$ relative to the line of sight. As the star rotates, the latter dependence leads to a rotational phase dependence of the spectra.

Models of partially ionized neutron-star atmospheres composed of strongly ionized iron, oxygen, and neon were constructed in $[67,68,83]$. The effects related to the finite nuclear masses were treated in the first order of the perturbation theory (see Sect. 2.2). The right panel of Fig. 1 shows local spectra of a strongly ionized carbon atmosphere at the magnetic pole and equator of a neutron star for the non-relativistic magnetic dipole model with $B_{\mathrm{p}}=2 \times 10^{12} \mathrm{G}$. It is clear that in this case the averaging over the surface, with spectra varying between these two extremes, would also result in smearing of some of the features seen in the local spectra.

The calculated spectra of partially ionized, strongly magnetized neutron star atmospheres composed of hydrogen, carbon, oxygen, and neon with magnetic fields $B \sim 10^{10}-10^{13} \mathrm{G}$ (see Sect. 2.4) are included in XSPEC [17] under the names NSMAX [68, 82] and NSMAXG [66, 68, 84], with the latter allowing for varying surface gravity. ${ }^{1}$

\subsection{Moderately strong magnetic fields}

Most of the thermally emitting INSs have surface magnetic fields in the range $10^{12} \mathrm{G} \lesssim B \lesssim$ $10^{15} \mathrm{G}$, but one class of sources, so called central compact objects (CCOs) have probably surface fields $B \lesssim 10^{11} \mathrm{G}$ [85-87]. These fields are strong enough to radically affect properties of hydrogen atoms and strongly quantize the electrons in the neutron-star atmosphere, but they are below the field strengths previously available in the atmosphere model NSMAXG [84]. The possibility to extend the model to the field strengths $3 \times 10^{10} \mathrm{G} \lesssim B \lesssim 10^{12} \mathrm{G}$, which we call moderately strong, has opened recently. The task of calculation of such models is arduous because the dimensionless field parameter $\gamma=B / B_{0}$ is smaller than in the previously calculated models, which entails the need to include more terms than previously in the wave-function expansion over the set of Landau orbitals [55]. In addition, with decreasing $B$, the energy spectrum of the bound states of a magnetized atom becomes denser, which necessitates inclusion of more such states in the consideration. Meanwhile, since $\gamma \gg 1$, the center-of mass motion of the atom noticeably affects the atomic properties.

In order to solve this problem, we constructed analytical fitting formulae for atomic energies, sizes, and main oscillator strengths as functions of $B$, discrete quantum numbers of initial and final states, and pseudomomentum $K_{\perp}$ [66]. For bound-free transitions, we calculated extensive tables of cross-sections as functions of $K_{\perp}$ and photon frequency $\omega$ for a number of bound states at every given $B$ and interpolated across these tables. This method allowed us to calculate the opacities for moderately magnetized hydrogen atmospheres [66]. In addition to the bound-bound, bound-free,

\footnotetext{
${ }^{1}$ We thank Peter Shternin for finding an error in the old (prior to 2014) version of NSMAX.
} 

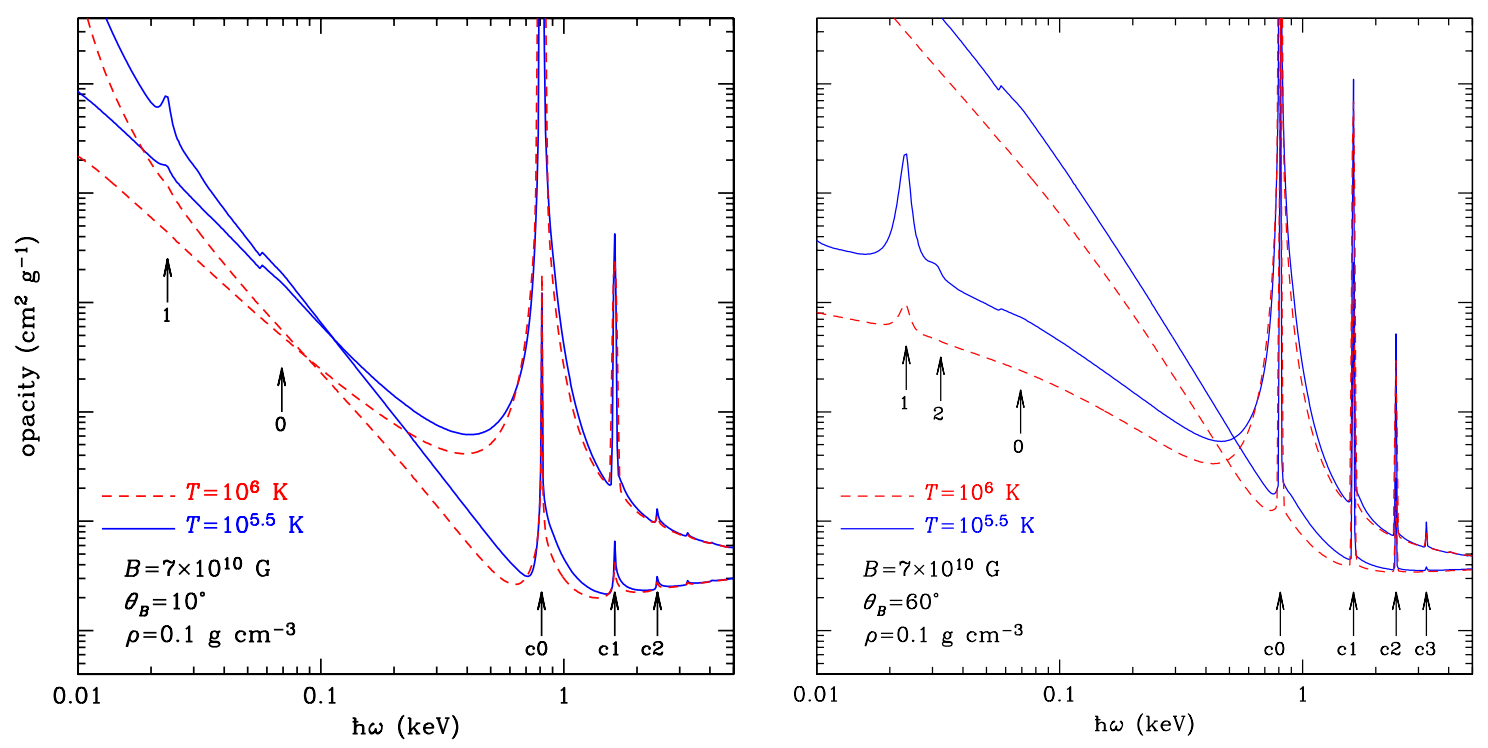

Figure 2: Opacities of the normal polarization modes $j=1,2$ (the lower and upper curve of each type) at $\rho=0.1 \mathrm{~g} \mathrm{~cm}^{-3}$ and $B=7 \times 10^{10} \mathrm{G}$ for $\theta_{k B}=10^{\circ}$ (left panel), $\theta_{k B}=60^{\circ}$ (right panel), $T=10^{5.5} \mathrm{~K}$ (solid curves, and $T=10^{6} \mathrm{~K}$ (dashed curves). The arrows marked $0,1,2$ correspond to different characteristic transition energies of the non-moving hydrogen atom: 0 - ground state binding energy, 1 - transition between the ground state and first excited state, 2 - transition between the first and second excited states. The arrows marked c $0, \mathrm{c} 1, \mathrm{c} 2, \mathrm{c} 3$ correspond to cyclotron harmonics energies $(N+1) \hbar \omega_{\mathrm{c}}$ with $N=0,1,2,3$, respectively.

and free-free absorption, we also included the cyclotron absorption with the electron cyclotron harmonics calculated beyond the cold plasma approximation (Sect. 2.2).

Examples of the calculated opacities are shown in Fig. 2 for two values of $\theta_{k B}$ and two values of $T$. Atomic features (marked $0,1,2$ ) are smeared and shifted leftward due to the magnetic broadening caused by the dependence of $E_{\mathrm{b}}$ on $K_{\perp}$, which is much larger than the usual Doppler broadening. At higher $T$, these features disappear because of the thermal ionization. At contrast, the electron cyclotron features (marked c0, c1, c2, c3) remain clearly visible even at high $T$. The calculated opacities have been archived at the Strasbourg astronomical Data Center. ${ }^{2}$

Work on the extension of the database NSMAXG to the moderately magnetized atmosphere models, applicable to CCOs, is in progress. Examples of the calculated spectra are shown in Fig. 3.

\section{Condensed surfaces and thin atmospheres}

Ruderman [88] suggested that a strong magnetic field can stabilize polymer chains directed along the field lines, and that the dipole-dipole attraction of these chains may result in a condensation. Later this conjecture was confirmed, although the binding and sublimation energies proved to be smaller than Ruderman assumed (see [89] and references therein).

From the thermodynamics point of view, the magnetic condensation is a phase transition caused by the strong electrostatic attraction between the ions and electrons in a dense plasma.

\footnotetext{
${ }^{2}$ http: //www.aanda.org/articles/aa/abs/2014/12/aa24619-14/aa24619-14.html
} 

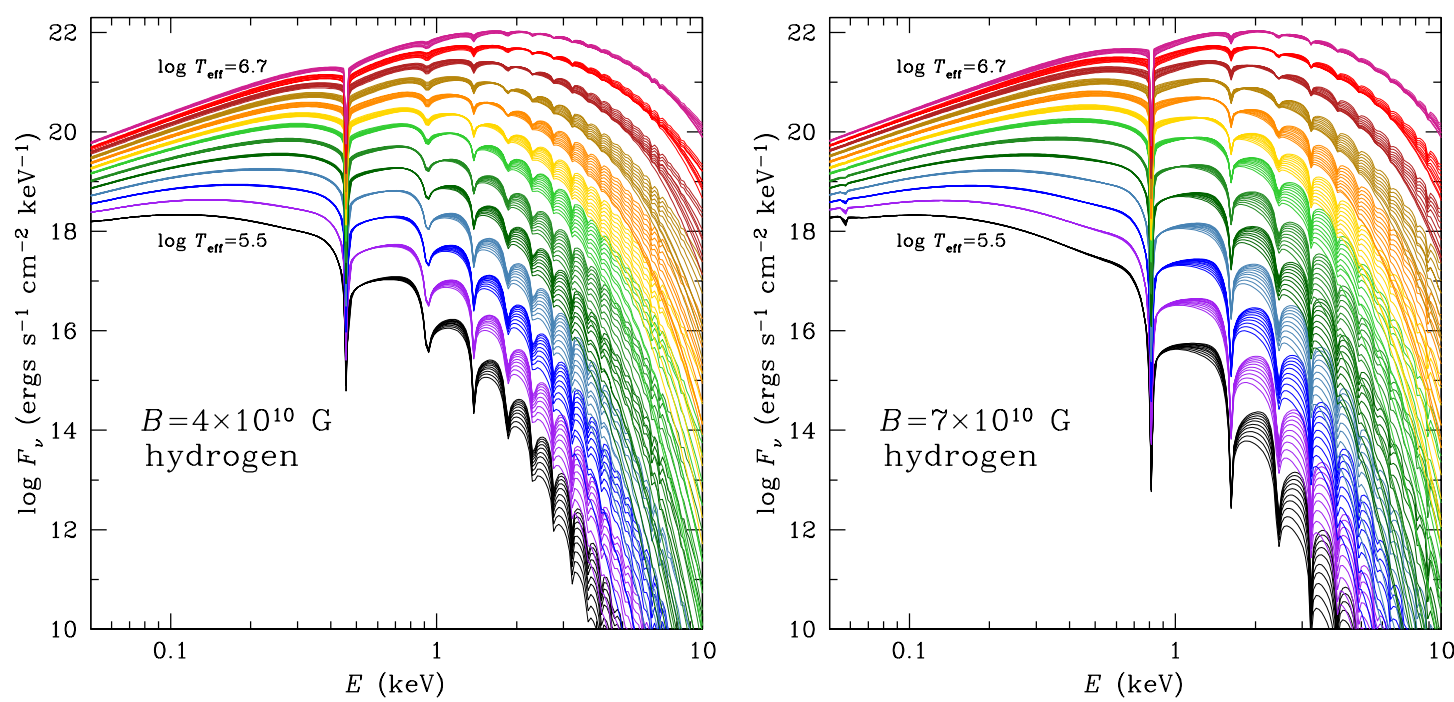

Figure 3: Spectra for partially ionized hydrogen atmosphere models with $B=4 \times 10^{10} \mathrm{G}$ (left panel) and $7 \times 10^{10} \mathrm{G}$ (right panel), with varying effective temperature $T_{\text {eff }}$ and surface gravity.

In the absence of magnetic field, such phase transitions were studied theoretically since 1930s (see [90] for a review). The simplest estimate of the phase transition domain is obtained in the ionsphere model [91], where the electrons are replaced by a uniform negative background, and the potential energy per ion is estimated as the electrostatic energy of the ionic interaction with the negative background contained in the sphere of radius $a_{\mathrm{i}}=\left(4 \pi n_{\mathrm{i}} / 3\right)^{-1 / 3}$, where $n_{\mathrm{i}}=n_{\mathrm{e}} / Z$ is the ion number density. At sufficiently high density, the electrostatic pressure is counterbalanced by the pressure of degenerate electrons. By equating the sum of the (negative) electrostatic pressure and the kinetic pressure of the electron gas to zero, one obtains density $\rho_{\mathrm{s}}$ of the condensed surface.

A strongly quantizing magnetic field lowers the electron Fermi temperature, therefore the above-mentioned pressure balance is shifted to higher densities. With increasing $\rho_{\mathrm{s}}$, the electrostatic attraction becomes stronger and, consequently, the critical temperature $T_{\text {crit }}$ of the phase transition also increases. For this reason, the phase transition may be expected in the neutron star envelopes despite their relatively high temperatures $T \sim\left(10^{5}-10^{7}\right) \mathrm{K}$. Lai [51] estimated the condensed-surface density as $\rho_{\mathrm{s}} \approx 561 \eta A Z^{-3 / 5} B_{12}^{6 / 5} \mathrm{~g} \mathrm{~cm}^{-3}$, where $\eta$ is an unknown factor, which would be equal to 1 in the ion sphere model. In more sophisticated models $\eta$ can differ from 1 because of ion correlations, electron-gas polarizability, and bound state formation. In particular, the values of $\rho_{\mathrm{s}}$ calculated by Medin \& Lai $[89,92]$ can be reproduced with $\eta=$ $0.517+0.24 / B_{12}^{1 / 5} \pm 0.011$ for carbon and $\eta=0.55 \pm 0.11$ for iron. The critical temperature $T_{\text {crit }}$, obtained numerically in $[89,92]$, is approximately (within a factor of 1.5 ) given by the expression $T_{\text {crit }} \approx 5 \times 10^{4} Z^{1 / 4} B_{12}^{3 / 4} \mathrm{~K}$ in the magnetic field range $10^{12} \mathrm{G} \lesssim B \lesssim 10^{15} \mathrm{G}$.

The thermal radiation of the condensed surface is determined by its emissivities in two normal modes, which are related to the reflectivities through the Kirchhoff law. Progressively improved calculations of the surface emissivity were presented in [93-99]. While the early works assumed that the ions are firmly fixed in the crystalline lattice, more recent works [97-99] consider also the 

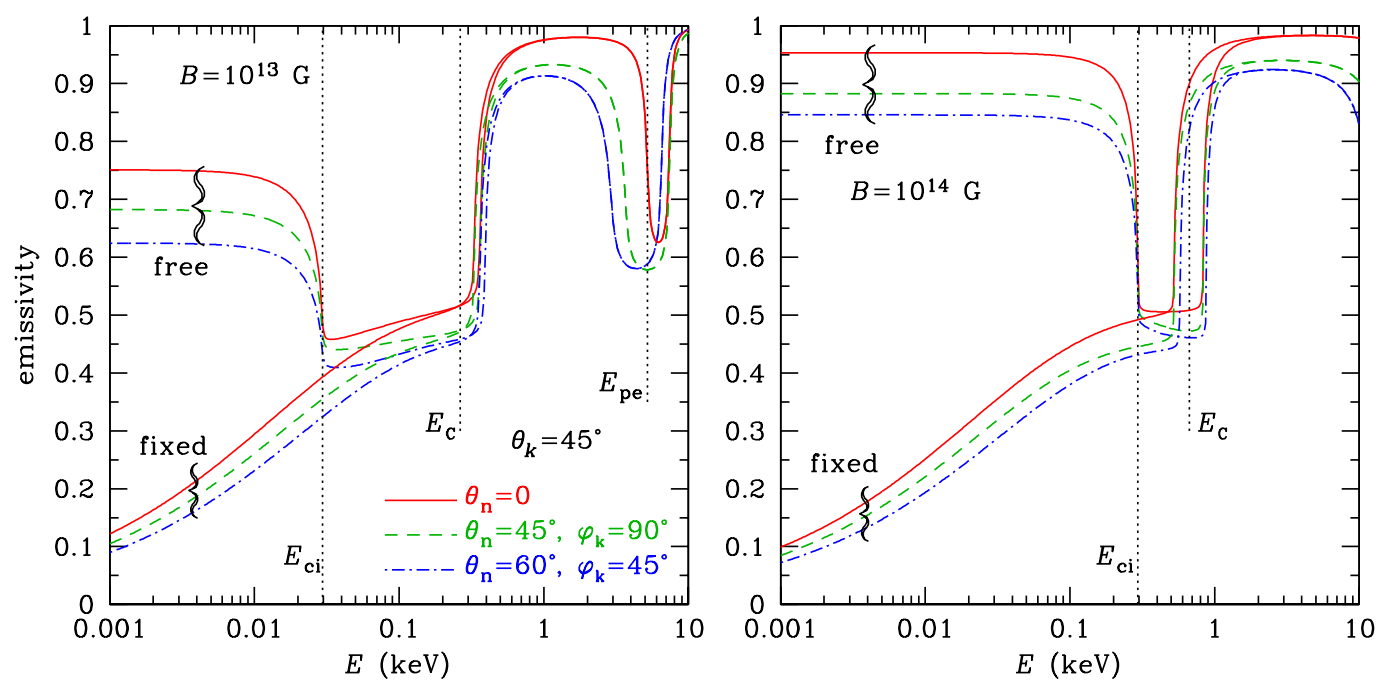

Figure 4: Dimensionless emissivity of a condensed iron surface at $B=10^{13} \mathrm{G}$ (left panel) and $10^{14} \mathrm{G}$ (right panel), averaged over polarizations, is shown as a function of energy of a photon emitted at the angle $\theta_{k}=45^{\circ}$, for different angles $\theta_{n B}$ between magnetic field $\boldsymbol{B}$ and the normal to the surface and angles $\varphi_{k}$ between the projections of $\boldsymbol{B}$ and wave vector $\boldsymbol{k}$ on the surface. For each parameter set, curves marked "free" and "fixed" correspond to models with free and fixed ions (see text). Vertical dotted lines mark positions of the characteristic energies: the ion cyclotron energy $E_{\mathrm{ci}}=\hbar \omega_{\mathrm{ci}}$, the electron plasma energy $E_{\mathrm{pe}}=\hbar \omega_{\mathrm{pe}}$, and the hybrid energy $E_{\mathrm{C}}$.

alternative model of free ions. The reality lies probably between these two extreme limits, but this problem has not yet been definitely solved.

The intensity of radiation from a condensed surface is equal to the Planck function $\mathscr{B}_{\omega, T}$ [Eq. (2.3)] multiplied by the normalized emissivity. Figure 4 shows examples of the normalized emissivity as a function of photon energy $E=\hbar \omega$, in both the free- and fixed-ion limits, for the wave-vector inclination angle $\theta_{k}=45^{\circ}$, for $B=10^{13} \mathrm{G}$ and $10^{14} \mathrm{G}$, and different values of the magnetic-field inclination $\theta_{n B}$ and azimuthal angles $\varphi_{k}$. The emissivity rapidly changes near the ion cyclotron energy $E_{\mathrm{ci}}=\hbar \omega_{\mathrm{ci}}$, the electron plasma energy $E_{\mathrm{pe}}=\hbar \omega_{\mathrm{pe}}$, and the energy $E_{\mathrm{C}}=$ $E_{\mathrm{ci}}+E_{\mathrm{pe}}^{2} / \hbar \omega_{\mathrm{c}}$ (see [98] for explanation of these features).

Motch et al. [100] suggested that some neutron stars can possess a hydrogen atmosphere of a finite thickness above the solid iron surface. If the optical depth of such atmosphere is small for some wavelengths and large for other ones, the thermal spectrum is different from that of thick atmospheres. Such spectra were calculated in [101-103] using simplified boundary conditions for the radiative transfer equation at the inner boundary of the atmosphere. More accurate boundary conditions [99] take into account that an extraordinary or ordinary wave, falling from the atmosphere on the surface, gives rise to reflected waves of both normal polarizations, whose intensities add to the respective intensities of the waves emitted by the condensed surface.

In general, local spectra of radiation emitted by thin hydrogen atmospheres over a condensed surface reveal a narrow absorption line corresponding to the proton cyclotron resonance in the atmosphere, features related to atomic transitions broadened by the motion effects (Sect. 2.2), and in the free-ions model also a kink corresponding to the ion cyclotron energy of the substrate ions. 
Some of these features may be absent, depending on the atmosphere thickness and magnetic field strength. At high energies, the spectrum is determined by the condensed-surface emission, which is softer than the spectrum of the thick hydrogen atmosphere.

One may also consider an atmosphere having a helium layer beneath the hydrogen layer. The spectrum of such "sandwich atmosphere" can have two or three absorption lines in the range $E \sim$ $(0.2-1) \mathrm{keV}$ at $B \sim 10^{14} \mathrm{G}[102]$.

\section{Polarization}

Thermal radiation emergent from neutron stars with strong magnetic fields is expected to be strongly polarized. Since the opacity is smaller for the extraordinary mode, with electric vector mainly transverse to the magnetic field, this mode escapes from deeper and hotter layers in the atmosphere, so that thermal radiation acquires polarization perpendicular to the local magnetic field [104]. Polarization of the observed radiation depends on the distribution of magnetic field and temperature over the visible neutron star surface. As the star rotates, the polarization pattern shows periodic variations, so that measuring the polarization pulse profile allows one to constrain the orientation of the rotation axis and the field strength and geometry [105, 106]. Therefore, future $\mathrm{X}$-ray polarization measurements are expected to resolve degeneracies that currently hamper the determination of magnetar physical parameters using thermal models [107, 108].

Polarization properties of the radiation can be described by the Stokes parameters $(I, Q, U, V)$, where $I$ is the total intensity, $Q$ and $U$ describe linear polarization, and $V$ circular polarization [109]. In terms of intensities $I_{\mathrm{O}}$ and $I_{\mathrm{X}}$ of the ordinary and extraordinary modes, $I=I_{\mathrm{X}}+I_{\mathrm{O}}$, $Q=\left(I_{\mathrm{O}}-I_{\mathrm{X}}\right) p_{\text {lin }} \cos 2 \phi, U=\left(I_{\mathrm{O}}-I_{\mathrm{X}}\right) p_{\text {lin }} \sin 2 \phi$, where $p_{\text {lin }}$ is the degree of linear polarization of each normal mode and $\phi$ is the angle between the major axis of the ordinary mode and the $x$-axis of the reference frame in which the Stokes parameters are defined [105, 110].

The thermal emission of a condensed surface is also polarized, because the reflectivities of normal modes are different. The polarization depends on the photon energy $E=\hbar \omega$ and angles $\theta_{n B}, \theta_{k}$, and $\varphi_{k}$ (Sect. 3). In a local spectrum, the degree of polarization can reach tens percent in some energy ranges. However, if radiation comes from a large surface area with varying magnetic field and temperature, the net polarization becomes much smaller.

After a photon has left the surface of a neutron star with a strong magnetic field, it travels through the magnetosphere and experiences the influence of vacuum polarization (see Sect. 2.1), which induces a change in the wave electric field as photon propagates. If the magnetic field is sufficiently strong, then in the vicinity of the star a typical length scale of this change is much shorter than the scale of the magnetic field; in this case the photon propagates adiabatically, so that its polarization instantaneously adapts to the variation of the magnetic field direction [111, 112]. Farther from the star the field decreases, and eventually photons leave the adiabatic region and maintain their polarization. The rays that leave the adiabatic region pass through only a small solid angle; consequently, polarizations of the rays originating in different regions will tend to align together. This effect partly lifts the strong suppression of the net polarization caused by the averaging over the field and temperature distributions at the star surface and can generate large net polarizations [113]. 

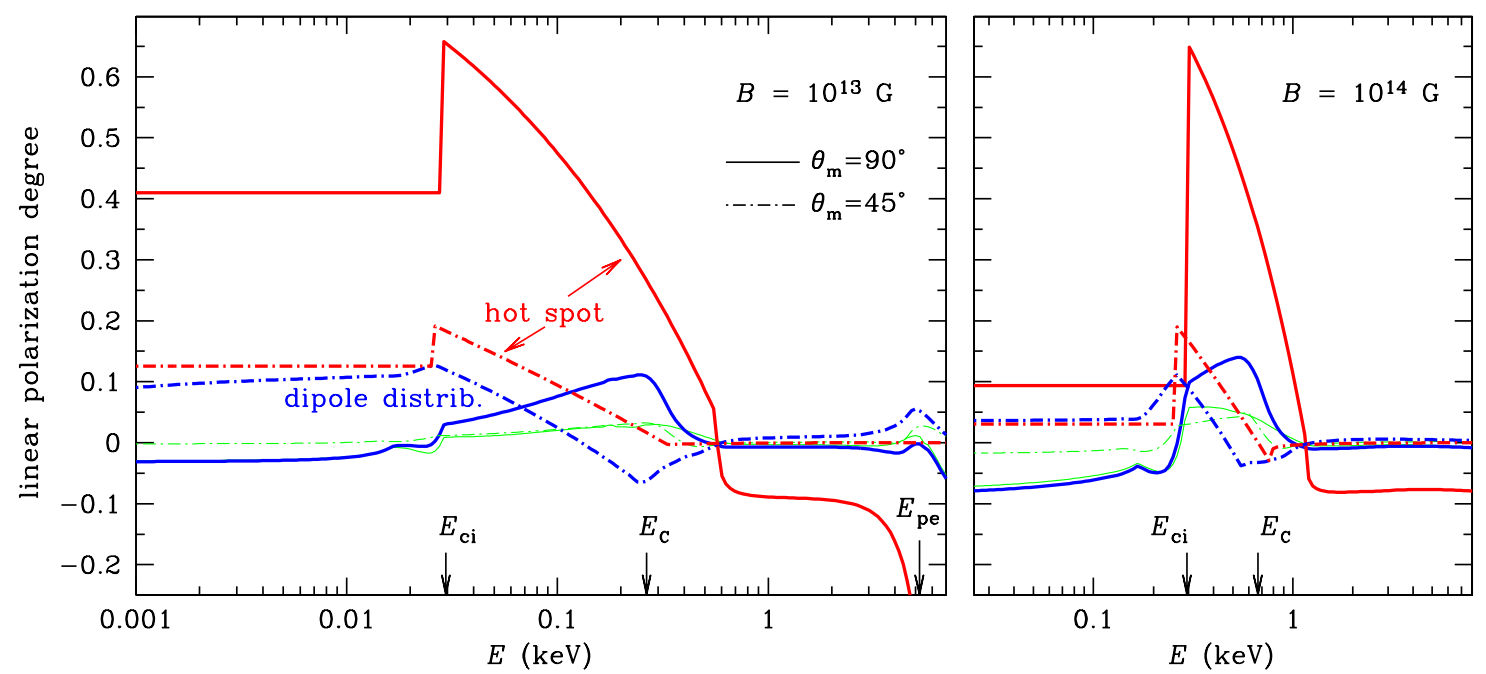

Figure 5: Average linear polarization (normalized Stokes parameter $Q / I$ in the reference frame where the $x$-axis is coplanar with the magnetic axis) is shown as a function of photon energy for the hot spot (red curves) or dipole field (blue curves), calculated in the model of free ions for a condensed magnetized iron surface, for two angles between the line of sight and magnetic axis $\theta_{\mathrm{m}}=90^{\circ}$ or $45^{\circ}$ (solid and dot-dashed lines, respectively) and the magnetic field at the pole (or in the hot spot) $B=10^{13} \mathrm{G}$ (left panel) or $10^{14} \mathrm{G}$ (right panel). In the case of the dipole model, the results obtained without allowance for the magnetospheric QED birefringence are shown by thin green lines for comparison. Energies $E_{\mathrm{ci}}, E_{\mathrm{C}}$, and $E_{\mathrm{pe}}$, marked at the horizontal axis, are the same as in Fig. 4.

A comparative analysis of the polarization of radiation from magnetized neutron stars with gaseous atmospheres and condensed surfaces was recently performed in [114]. The authors proposed a simplified treatment of photon propagation through the adiabatic region. Instead of integrating the equations that govern the photon polarization from the neutron star surface to the infinity, the propagation is assumed perfectly adiabatic (i.e., each photon preserves its polarization with respect to the local magnetic field direction) up to some radius, where the typical scales of variations of the photon electric field and external magnetic field become comparable, and the change of polarization at larger distances is neglected. This adiabatic radius is estimated as $r_{\mathrm{ad}}=4.8\left(B / 10^{11} \mathrm{G}\right)^{2 / 5}(E / 1 \mathrm{keV})^{1 / 5} R$.

Figure 5 shows the linear polarization degree in the model of free ions for the two cases: first, where the radiation spectrum is formed at a hot spot with $\boldsymbol{B}$ normal to the surface, and second, where the field is distributed according to the relativistic dipole model. In the latter case, an isotropic internal (core) temperature of $10^{8} \mathrm{~K}$ is assumed and a surface temperature distribution is calculated according to Ref. [75] (this calculation gives $T_{\text {eff }} \approx 1.0 \mathrm{MK}$ for $B_{\mathrm{p}}=10^{13} \mathrm{G}$ and $T_{\text {eff }} \approx 1.3 \mathrm{MK}$ for $B_{\mathrm{p}}=10^{14} \mathrm{G}$ ). The figure illustrates the polarization smearing away by the averaging over the stellar surface and the opposite effect due to the adiabatic propagation of radiation in the near zone of the star. 


\section{Theory versus observations}

As argued above, models of strongly magnetized $\left(B \gg 10^{9} \mathrm{G}\right)$ neutron-star atmospheres must take the bound species and their radiative transitions into account. Let us consider a few examples where models of magnetized, partially ionized atmospheres have been used to study their thermal radiation.

\subsection{RX J1856.5-3754}

RX J1856.5-3754 is the closest and brightest of the class of X-ray (dim) INSs (XINSs or XDINS, also known as the Magnificent Seven), whose X-ray spectra are apparently of purely thermal nature (see [115] for a review). The spectra of the other XINSs show broad absorption features at several hundred $\mathrm{eV}$, and at least four of them also exhibit narrow absorption features [116]. RX J1856.5-3754 is the only one of the Seven that has a blackbody-like X-ray spectrum without absorption features. Similar to the other XINSs, its X-ray and optical spectra correspond to substantially different effective temperatures if fitted separately with blackbodies. However, the measured spectrum was fitted in the entire range from X-rays to optical within observational errorbars with the use of the model of a thin magnetized hydrogen atmosphere on top of a condensed iron surface [101]. The best agreement between the theoretical and observed spectra has been achieved for the atmosphere column density $y_{\mathrm{col}}=1.2 \mathrm{~g} \mathrm{~cm}^{-2}$, gravitational redshift $z_{g} \approx 0.25 \pm 0.05$, and apparent radius $R_{\infty}=17.2_{-0.1}^{+0.6} D_{140} \mathrm{~km}$, where $D_{140}$ is distance in units of $140 \mathrm{pc}$. The authors used a piecewise approximation to dipole-like distributions of the magnetic field and temperature with the polar values $B_{\mathrm{p}} \sim 6 \times 10^{12} \mathrm{G}, T_{\mathrm{s}} \sim 0.7 \mathrm{MK}$, whereas the overall effective temperature is $T_{\text {eff }}^{\infty}=0.434 \pm 0.003 \mathrm{MK}(1 \sigma$ significance $)$. Using an updated distance estimate, $D=123_{-15}^{+11} \mathrm{pc}$ [117], the above estimates of $z_{g}$ and $R_{\infty}$ give $R=12.1_{-1.6}^{+1.3} \mathrm{~km}$ and $M=1.5 \pm 0.3 M_{\odot}$. Note that a fit of the observed X-ray spectrum with the Planck function yields a $70 \%$ higher temperature and a 3.5 times smaller radius of the emitting surface. Such huge difference exposes the importance of a correct physical interpretation of an observed spectrum for evaluation of neutron star parameters (as well as in the non-magnetic thermally emitting INSs; cf. Ref. [118]).

The inferred magnetic field is significantly smaller than $B_{\mathrm{s}} \approx 1.5 \times 10^{13} \mathrm{G}$ obtained from timing analysis [119]. Here, $B_{\mathrm{S}}$ is so-called spindown or characteristic magnetic field, that is the value of the equatorial magnetic field in the "canonical model" of a rotating non-relativistic magnetic dipole in vacuum [120], with the rotation axis orthogonal to the magnetic axis, with radius $R=10$ $\mathrm{km}$, and with moment of inertia $I=10^{45} \mathrm{~g} \mathrm{~cm}^{2}$. Assuming different $R$ and $I$, one would obtain another field estimate, proportional to $\sqrt{I} / R^{3}$. For instance, modern BSk21 equation of state for the neutron star matter (Ref. [121] and references therein) yields $R=12.6 \mathrm{~km}$ and $I=2.06 \times 10^{45}$ $\mathrm{g} \mathrm{cm}^{2}$ for $M=1.5 M_{\odot}$, which gives a factor 0.7 to the canonical $B_{\mathrm{s}}$ estimate. Still more realistic estimate should take into account that a real neutron star is surrounded by magnetosphere, and the angle $\xi$ between its rotational and magnetic axes can differ from $90^{\circ}$. An analytic fit to the results of numerical simulations presented in Ref. [122] gives a correction factor $\approx 0.8 /\left(1+\sin ^{2} \xi\right.$ ) to the canonical estimate of $B$. Taken together, the two factors translate the result of Ref. [119] into $B \sim(4-8) \times 10^{12} \mathrm{G}$. On the other hand, an alternative interpretation of the spindown in terms of the fallback disk model leads to still smaller estimate $B \sim 10^{12} \mathrm{G}$ [123]. 
Analysis [124] of the light curve of RX J1856.5-3754 with the aid of the same thin-atmosphere model yields constraints on the geometry of rotational and magnetic axes. It turned out that the light curve can be explained if one of these angles is small $\left(<6^{\circ}\right)$, while the other angle lies between $20^{\circ}$ and $45^{\circ}$. In this case, the radio emission around the magnetic poles does not cross the line of sight. As noted in [124], this may explain the non-detection of this star as a radio pulsar [125].

\subsection{RX J1308.6+2127}

Hambaryan et al. [126] described the X-ray spectrum of RX J1308.6+2127 (RBS 1223) by a wide absorption line centered around $\hbar \omega=0.3 \mathrm{keV}$, superposed on the Planck spectrum, with the line parameters depending on the stellar rotation phase. This source shows the highest pulsed fraction (13-42\%, depending on energy band) of all the XINSs. A phase resolved spectrum obtained from all 2003-2007 XMM-Newton observations of this star has been reproduced by the model a magnetized iron surface covered by a partially ionized hydrogen atmosphere with $y_{\text {col }} \sim 1-$ $10 \mathrm{~g} \mathrm{~cm}^{-2}$, with mutually consistent asymmetric bipolar distributions of the magnetic field and the temperature, with the polar values $B_{\mathrm{p} 1}=B_{\mathrm{p} 2}=(0.86 \pm 0.02) \times 10^{14} \mathrm{G}, T_{\mathrm{p} 1}=1.22_{-0.05}^{+0.02} \mathrm{MK}$, and $T_{\mathrm{p} 2}=1.15 \pm 0.04 \mathrm{MK}$. The effective temperature is $T_{\mathrm{eff}} \approx 0.7 \mathrm{MK}$. The gravitational redshift is estimated to be $z_{g}=0.16_{-0.01}^{+0.03}$, which converts into $\left(M / M_{\odot}\right) / R_{6}=0.87_{-0.05}^{+0.13}$ and suggests a stiff EOS of the neutron star matter.

\subsection{E 1207.4-5209}

The discovery of absorption lines by $[127,128]$ in the spectrum of CCO 1E $1207.4-5209$ at energies $E \sim 0.7 N \mathrm{keV}(N=1,2, \ldots)$ immediately led to the belief that they are caused by cyclotron harmonics. As we mentioned in Sect. 2.2, such harmonics could be only electronic, because the ion cyclotron harmonics are unobservable. Therefore, this interpretation implies $B \approx 7 \times 10^{10} \mathrm{G}$. Initially this interpretation was criticized because the relative strengths of the spectral absorptions caused by the cyclotron fundamental and harmonics were thought to be sharply different, but this argument was based on the considerations in the cold plasma approximation and does not hold beyond it [45] (as confirmed by our Fig. 3).

There are doubts concerning the statistical significance of the third and fourth observed lines [129], while the first and second lines were tentatively interpreted as characteristic features in an oxygen atmosphere with magnetic field $B \approx 10^{12} \mathrm{G}$ [67]. However, recent timing analysis [130] gives the characteristic field $B_{\mathrm{s}}=9.8 \times 10^{10} \mathrm{G}$ and thus favors the electron-cyclotron interpretation.

\subsection{XMM J104608.7-594306}

2XMM J104608.7-594306 is a thermally emitting INS, but unlike the Magnificent Seven it has a short spin period of only $18.6 \mathrm{~ms}$. Its XMM-Newton spectrum has been analyzed in [131], using the blackbody model and hydrogen atmosphere models NSA and NSMAXG. Statistically acceptable spectral fits and meaningful physical parameters for the source are only obtained when the purely thermal spectrum is modified by absorption lines at $E \approx 0.55 \mathrm{keV}$ and $1.3 \mathrm{keV}$. The authors fixed $M$ to $1.4 M_{\odot}$ and tried various values of magnetic field from 0 to $3 \times 10^{13} \mathrm{G}$. In the case of the NSMAXG model, the best statistical significance $\left(\chi^{2} /\right.$ d.o.f. $\left.<1.1\right)$ was provided by the assumptions of $B=10^{10} \mathrm{G}$ and $B=3 \times 10^{13} \mathrm{G}$. In the former case, the inferred apparent radius $R_{\infty}=9 \mathrm{~km}$ 
is implausibly small, whereas in the latter case $R_{\infty}=12 \mathrm{~km}$ does not contradict to theoretical neutron star models. In the latter case, the inferred effective temperature is $T_{\text {eff }}=0.86_{-0.04}^{+0.07} \mathrm{MK}$ (for comparison, the blackbody model gives the redshifted temperature $T_{\mathrm{eff}}^{\infty} \sim(1.6-1.9) \mathrm{MK}$ and $\left.R_{\infty} \sim 1.5-3 \mathrm{~km}\right)$.

\subsection{WGA J1952.2+2925}

1WGA J1952.2+2925 is the central X-ray source in the pulsar wind nebula DA 495, but it shows no pulsar activity. An analysis [132] of its archival Chandra and XMM-Newton data shows that it has a pure thermal spectrum which is equally well fitted either by the blackbody model with a temperature of $k_{\mathrm{B}} T \approx 215 \mathrm{eV}$ and an emitting area radius of $\approx 0.6 \mathrm{~km}$ or by magnetized neutron star atmosphere model NSMAX with $k_{\mathrm{B}} T_{\text {eff }} \sim 80-90 \mathrm{eV}$. In the latter case the thermal emission can come from the entire neutron star surface. The authors [132] also placed an upper limit on the non-thermal flux and an upper limit of $40 \%$ on the pulsed fraction. The authors suggested that the Fermi source 3FGL J1951.6+2926 is the likely $\gamma$-ray counterpart of 1WGA J1952.2+2925.

\subsection{Rotation powered pulsars: PSR J1119-6127, B0943+10, J0357+3205, and J0633+0632}

The partially ionized, strongly magnetized hydrogen atmosphere model NSMAX (Sect. 2.3) was applied in [133] to interpret observations of pulsar J1119-6127, for which the estimate based on spindown gives an atypically high characteristic field $B_{\mathrm{s}}=4 \times 10^{13} \mathrm{G}$. In the X-ray range, it emits pulsed radiation, with a significant thermal component. At fixed $D=8.4 \mathrm{kpc}$ and $R=13$ $\mathrm{km}$, the bolometric flux gives $T_{\text {eff }} \approx 1.1 \mathrm{MK}$. It was difficult to explain, however, the large pulsed fraction ( $48 \pm 12 \%)$ by the thermal emission. $\mathrm{Ng}$ et al. [133] managed to reproduce the X-ray light curve of this pulsar assuming that one of its magnetic poles is surrounded by an area, which is covered by hydrogen and heated to $1.5 \mathrm{MK}$, while the temperature of the opposite polar cap is below $0.9 \mathrm{MK}$.

A similar analysis was applied in [134] to interpret observations of pulsar B0943+10, which show correlated radio and $\mathrm{X}$-ray mode switches. The authors take $B=2 \times 10^{12} \mathrm{G}$ inferred from the pulsar spindown, assumed $M=1.2 M_{\odot}$ and $R=12 \mathrm{~km}$, and modeled the emitting area as a hot spot covered by a partially ionized hydrogen atmosphere. They find that an atmosphere with $T_{\text {eff }} \approx(1.4-1.5) \mathrm{MK}$ and emission radius $R_{\mathrm{em}} \approx 85 \mathrm{~m}$ matches the radio-quiet $\mathrm{X}$-ray spectrum, whereas previous blackbody fits gave temperature $\approx 3 \mathrm{MK}$ and emission radius $\approx 20-30 \mathrm{~m}$. The authors show that the large X-ray pulse fraction observed during the radio quiet phase can be explained by including the beaming effect of a magnetic atmosphere.

PSR J0357+3205 is an unusual thermally-emitting pulsar observed in X-rays. Its spectrum was fitted in [135] with several different multicomponent models. In the physically realistic case where the incomplete ionization of the atmosphere was taken into account, the authors used the NSMAX model for the thermal spectral component and a power-law model for the non-thermal one and fixed $M=1.4 M_{\odot}$ and $B=10^{12} \mathrm{G}$. They obtained an acceptable fit $\left(\chi^{2} /\right.$ d.o.f $\left.=1.05\right)$ with a loose constraint on the radius, $R_{\infty}=8_{-5}^{+12}(D / 500 \mathrm{pc}) \mathrm{km}$.

Danilenko et al. [136] analyzed Chandra observations of the bright Fermi pulsar J0633+0632 and confirmed early findings that its X-ray spectrum contains non-thermal and thermal components. The latter is equally well described by the blackbody model and magnetized atmosphere model 
NSMAX and can be attributed to the emission from the bulk of the stellar surface. In the latter case $k_{\mathrm{B}} T_{\text {eff }}=53_{-7}^{+12} \mathrm{eV}$ (at $90 \%$ confidence), which makes this pulsar one of the coldest middle-aged INSs with measured temperatures. The authors also reported evidence of an absorption feature at $E=804_{-26}^{+42} \mathrm{eV}$ with equivalent width of $63_{-36}^{+47} \mathrm{eV}$.

\section{Conclusions}

We have considered the main features of neutron-star atmospheres and radiating surfaces and outlined the current state of the theory of the formation of their spectra (see [23] for more details). The interpretation of observations enters a qualitatively new phase, free from the assumptions of a blackbody spectrum or the "canonical model" of neutron stars. Spectral features, compatible with absorption lines in some cases, have been discovered in thermal spectra of strongly magnetized neutron stars. On the agenda is their detailed theoretical description, which may provide information on the surface composition, temperature and magnetic field distributions. However, in order to disentangle these parameters, a number of problems related to the theory of magnetic atmospheres and radiating surfaces still have to be solved.

Acknowledgements. A.Y.P. is grateful to the organizers of the conference "The Modern Physics of Compact Stars 2015" for the invitation, hospitality, and financial support of participation. We thank the referee, Valery Suleimanov for careful reading the manuscript and useful remarks. The work of A.Y.P. has been partly supported by the RFBR (grant 14-02-00868). W.C.G.H. appreciates use of computer facilities at KIPAC.

\section{References}

[1] S.Tsuruta, Neutron Star Models (Ph.D. Thesis). Columbia Univ., New York (1964)

[2] H.-Y. Chiu, Supernovae, neutrinos, and neutron stars, Ann. Phys. (NY) 26 (1964) 364

[3] R. Giacconi, H. Gursky, F.R. Paolini, B.B. Rossi, Evidence for X-rays from sources outside the solar system, Phys. Rev. Lett. 9 (1962) 439

[4] S. Bowyer, E.T. Byram, T.A. Chubb, H. Friedman, X-ray sources in the Galaxy, Nature 201 (1964) 1307

[5] S. Bowyer, E.T. Byram, T.A. Chubb, H. Friedman, Lunar occultation of X-ray emission from the Crab nebula, Science 146 (1964) 912

[6] A. Sandage, P. Osmer, R. Giacconi, et al., On the optical identification of Sco X-1, Astrophys. J. 146 (1966) 316

[7] I.S. Shklovsky, On the nature of the source of X-ray emission of Sco XR-1, Astrophys. J. 148 (1967) L1

[8] A.G.W. Cameron, Stellar accretion and X-ray emission, Nature 215 (1967) 464

[9] J.A. de Freitas Pacheco, J.E. Steiner, A.D. Neto, The electromagnetic emission from Sco X-1, Astron. Astrophys. 55 (1977) 111

[10] A.F. Cheng \& D.J. Helfand, X-rays from radio pulsars - The detection of PSR 1055-52, Astrophys. J. 271 (1983) 271 
[11] W. Brinkmann \& H. Ögelman, Soft X-ray observations of the radio pulsar PSR 1055-52, Astron. Astrophys. 182 (1987) 71

[12] H. Ögelman, X-ray observations of cooling neutron stars, in proceedings of the NATO ASI conference The Lives of the Neutron Stars, ed. by M.A. Alpar, Ü. Kiziloğlu, J. van Paradijs, NATO ASI Ser. C 450. Kluwer, Dordrecht (1995) 101

[13] W. Becker, An X-ray study of isolated neutron stars and their wind nebulae, in Highlights in X-ray Astronomy, ed. by B. Aschenbach \& M.J. Freyberg, MPE Report 272. MPE, Garching (1999), 49

[14] V.E. Zavlin, G.G. Pavlov, Yu.A. Shibanov, Model neutron star atmospheres with low magnetic fields. I. Atmospheres in radiative equilibrium, Astron. Astrophys. 315 (1996) 141 [arXiv:astro-ph/9604072]

[15] B.T. Gänsicke, T.M. Braje, R.M. Romani, Thermal emission from low-field neutron stars, Astron. Astrophys. 386 (2002) 1001 [arXiv:astro-ph/0110130]

[16] C.O. Heinke, G.B. Rybicki, R. Narayan, J.E. Grindlay, A hydrogen atmosphere spectral model applied to the neutron star X7 in the globular cluster 47 Tucanae, Astrophys. J. 644 (2006) 1090 [arXiv:astro-ph/0506563]

[17] K.A. Arnaud, XSPEC: the first ten years, in Astronomical Data Analysis Software and Systems V, ed. by G. Jacoby, J. Barnes, ASP Conf. Ser. 101 (1996) 17; [http://starchild.gsfc.nasa.gov/xanadu/xspec/]

[18] C.B. Haakonsen, M.L. Turner, N.A. Tacik, R.E. Rutledge, McPHAC: McGill Planar Hydrogen Atmosphere Code, Astrophys. J. 749 (2012) 52 [https://github.com/McP HAC/]

[19] M. Rajagopal \& R. Romani, Model atmospheres for low-field neutron stars, Astrophys. J. 461 (1996) 327 [arXiv:astro-ph/9510094]

[20] J.A. Pons, F.M. Walter, J.M. Lattimer, et al., Toward a mass and radius determination of the nearby isolated neutron star RX J185635-3754, Astrophys. J. 564 (2002) 981 [arXiv:astro-ph/0107404]

[21] W.C.G. Ho \& C.O. Heinke, A neutron star with a carbon atmosphere in the Cassiopeia A supernova remnant, Nature 462 (2009) 71 [arXiv : 0911 . 0672]

[22] V.F. Suleimanov, D. Klochkov, G.G. Pavlov, K. Werner, Carbon neutron star atmospheres, Astrophys. J. Suppl. Ser. 210 (2014) 13 [arXiv:1311. 6037]

[23] A.Y. Potekhin, Atmospheres and radiating surfaces of neutron stars, Phys. Usp. 57 (2014) 735 [arXiv: 1403.0074$]$

[24] V.F. Suleimanov, J. Poutanen, D. Klochkov, K. Werner, Measuring the basic parameters of neutron stars using model atmospheres, Eur. Phys. J. A 52 (2016) 20 [arXiv:1510.06962]

[25] S.A. Olausen, V.M. Kaspi, The McGill Magnetar Catalog, Astrophys. J. Suppl. Ser. 212 (2014) 6 [arXiv:1309.4167]

[26] S. Mereghetti, J.A. Pons, A. Melatos, Magnetars: properties, origin and evolution, Space Sci. 
Rev. 191 (2015) 315 [arXiv: 1503.06313 ]

[27] R. Turolla, S. Zane, A.L. Watts, Magnetars: the physics behind observations. A review, Rep. Prog. Phys. 78 (2015) 116901 [arXiv: 1507.02924 ]

[28] Yu.N. Gnedin \& G.G. Pavlov, The transfer equations for normal waves and radiation polarization in an anisotropic medium, Sov. Phys. JETP 38 (1974) 903

[29] V.L. Ginzburg, The Propagation of Electromagnetic Waves in Plasmas (2nd ed.). Pergamon, London (1970)

[30] A.D. Kaminker, G.G. Pavlov, Yu.A. Shibanov, Radiation for a strongly-magnetized plasmaThe case of predominant scattering, Astrophys. Space Sci. 86 (1982) 249

[31] V.V. Zheleznyakov, Radiation in Astrophysical Plasmas. Kluwer, Dordrecht (1996)

[32] V. Suleimanov, J. Poutanen, K. Werner, X-ray bursting neutron star atmosphere models using an exact relativistic kinetic equation for Compton scattering, Astron. Astrophys. 545 (2012) A120 [arXiv:1208.1467]

[33] T. Bulik \& G.G. Pavlov, Polarization modes in a strongly magnetized hydrogen gas, Astrophys. J. 469 (1996) 373 [arXiv: astro-ph/9512081]

[34] A.Y. Potekhin, D. Lai, G. Chabrier, W.C.G. Ho, Electromagnetic polarization in partially ionized plasmas with strong magnetic fields and neutron star atmosphere models, Astrophys. J. 612 (2004) 1034 [arXiv: astro-ph/ 0405383 ]

[35] G.G. Pavlov \& Yu.N. Gnedin, Vacuum polarization by a magnetic field and its astrophysical manifestations, Sov. Sci. Rev. E: Astrophys. Space Phys. 3 (1984) 197

[36] S.L. Adler, Photon splitting and photon dispersion in a strong magnetic field, Ann. Phys. (NY) 67 (1971) 599

[37] J.S. Heyl \& L. Hernquist, Analytic form for the effective Lagrangian of QED and its application to pair production and photon splitting, Phys. Rev. D 55 (1997) 2449 [arXiv:hep-th/9607124]

[38] K. Kohri \& S. Yamada, Polarization tensors in strong magnetic fields, Phys. Rev. D 65 (2002) 043006 [arXiv: astro-ph/0102225]

[39] W.C.G. Ho \& D. Lai, Atmospheres and spectra of strongly magnetized neutron stars - II. The effect of vacuum polarization, Mon. Not. R. Astron. Soc. 338 (2003) 233 [arXiv:astro-ph/0201380]

[40] Yu.A. Shibanov \& V.E. Zavlin, Calculations of the photospheres of magnetic neutron stars, Astron. Lett. 21 (1995) 3

[41] A.A. Sokolov \& I.M. Ternov, Radiation from Relativistic Electrons. Am. Inst. Phys., New York (1986)

[42] P. Mészáros, High-Energy Radiation from Magnetized Neutron Stars. University of Chicago Press, Chicago (1992)

[43] J. Ventura, Scattering of light in a strongly magnetized plasma, Phys. Rev. D 19 (1979) 1684

[44] G.G. Pavlov, Yu.A. Shibanov, D.G. Yakovlev, Quantum effects in cyclotron plasma absorp- 
tion, Astrophys. Space Sci. 73 (1980) 33

[45] V.F. Suleimanov, G.G. Pavlov, K. Werner, Magnetized neutron star atmospheres: beyond the cold plasma approximation, Astrophys. J. 751 (2012) 15 [arXiv:1201.5527]

[46] G.G. Pavlov \& A.N. Panov, Photon absorption and emission in Coulomb collisions in a magnetic field, Sov. Phys. JETP 44 (1976) 300

[47] A.Y. Potekhin \& G. Chabrier, Equation of state and opacities for hydrogen atmospheres of neutron stars with strong magnetic fields, Astrophys. J. 585 (2003) 955 [arXiv:astro-ph/0212062]

[48] A.Y. Potekhin, Cyclotron harmonics in opacities of isolated neutron star atmospheres, Astron. Astrophys. 518 (2010) A24 [arXiv:1003.5106]

[49] R. Cohen, J. Lodenquai, M. Ruderman, Atoms in superstrong magnetic fields, Phys. Rev. Lett. 25 (1970) 467

[50] H. Ruder, G. Wunner, H. Herold, F. Geyer, Atoms in Strong Magnetic Fields. Springer, Berlin (1994)

[51] D. Lai, Matter in strong magnetic fields, Rev. Mod. Phys. 73 (2001) 629 [arXiv:astro-ph/0009333]

[52] B.R. Johnson, J.O. Hirschfelder, K.H. Yang, Interaction of atoms, molecules and ions with constant electric and magnetic fields, Rev. Mod. Phys. 55 (1983) 109

[53] L.P. Gor'kov \& I.E. Dzyaloshinskiŭ, Contribution to the theory of the Mott exciton in a strong magnetic field, Sov. Phys. JETP 26 (1968) 449

[54] M. Vincke, M. Le Dourneuf, D. Baye, J. Phys. B: At. Mol. Phys. 25 (1992) 2787

[55] A.Y. Potekhin, Structure and radiative transitions of the hydrogen atom moving in a strong magnetic field, J. Phys. B: At. Mol. Opt. Phys. 27 (1994) 1073

[56] G.G. Pavlov \& A.Y. Potekhin, Bound-bound transitions in strongly magnetized hydrogen plasma, Astrophys. J. 450 (1995) 883

[57] A.Y. Potekhin \& G.G. Pavlov, Photoionization of hydrogen in atmospheres of magnetic neutron stars, Astrophys. J. 483 (1997) 414 [arXiv: astro-ph/9702004]

[58] V.G. Bezchastnov, G.G. Pavlov, J. Ventura, Discrete eigenstates of the $\mathrm{He}^{+}$ion moving in a strong magnetic field, Phys. Rev. A 58 (1998) 180

[59] G.G. Pavlov \& V.G. Bezchastnov, Once-ionized helium in superstrong magnetic fields, Astrophys. J. 635 (2005) L61 [arXiv: astro-ph/0505464]

[60] M. Vincke \& D. Baye, Centre-of-mass effects on the hydrogen atom in a magnetic field, J. Phys. B: At. Mol. Phys. 21 (1988) 2407

[61] G.G. Pavlov \& P. Mészáros, Finite-velocity effects on atoms in strong magnetic fields and implications for neutron star atmospheres, Astrophys. J. 416 (1993) 752

[62] K. Mori \& C.J. Hailey, Atomic calculation for the atmospheres of strongly magnetized neutron stars, Astrophys. J. 564 (2002) 914 [arXiv:astro-ph/0109214] 
[63] A.Y. Potekhin, G. Chabrier, Yu.A. Shibanov, Partially ionized hydrogen plasma in strong magnetic fields, Phys. Rev. E 60 (1999) 2193 [arXiv:astro-ph/9907006]

[64] K. Mori \& J. Heyl, Ionization and dissociation equilibrium in strongly magnetized helium atmosphere, Mon. Not. R. Astron. Soc. 376 (2007) 895 [arXiv: astro-ph/ 0610253 ]

[65] A.Y. Potekhin \& G. Chabrier, Equation of state and opacities for hydrogen atmospheres of magnetars, Astrophys. J. 600 (2004) 317 [arXiv: astro-ph/ 0309310 ]

[66] A.Y. Potekhin, G. Chabrier, W.C.G. Ho, Opacities and spectra of hydrogen atmospheres of moderately magnetized neutron stars Astron. Astrophys. 572 (2014) A69 [arXiv:1409.7651]

[67] K. Mori \& C.J. Hailey, Detailed atmosphere modeling for the neutron star 1E1207.4-5209: Evidence of oxygen/neon atmosphere, Astrophys. J. 648 (2006) 1139 [arXiv:astro-ph/0301161]

[68] K. Mori, W.C.G. Ho, Modelling mid-Z element atmospheres for strongly magnetized neutron stars, Mon. Not. R. Astron. Soc. 377 (2007) 905 [arXiv: astro-ph/ 0611145 ]

[69] G. Greenstein \& G.J. Hartke, Pulselike character of blackbody radiation from neutron stars, Astrophys. J. 271 (1983) 283

[70] A.M. Beloborodov, Gravitational bending of light near compact objects, Astrophys. J. 566 (2002) L85 [arXiv:astro-ph/0201117]

[71] J. Poutanen \& A.M. Beloborodov, Pulse profiles of millisecond pulsars and their Fourier amplitudes, Mon. Not. R. Astron. Soc. 373 (2006) 836 [arXiv: astro-ph / 0608663 ]

[72] V.L. Ginzburg \& L.M. Ozernoi, On gravitational collapse of magnetic stars, Sov. Phys. JETP 20 (1965) 689

[73] D. Viganò, N. Rea, J.A. Pons, R. Perna, D.N. Aguilera, J.A. Miralles, Unifying the observational diversity of isolated neutron stars via magneto-thermal evolution models, Mon. Not. $R$. Astron. Soc. 434 (2013) 123 [arXiv: 1306.2156 ]

[74] J.G. Elfritz, J.A. Pons, N. Rea, K. Glampedakis, D. Viganò, Simulated magnetic field expulsion in neutron star cores, Mon. Not. R. Astron. Soc. 456 (2016) 4461 [arXiv:1512.07151]

[75] A.Y. Potekhin, J.A. Pons, D. Page, Neutron stars - Cooling and transport, Space Sci. Rev 191 (2015) 239 [arXiv: 1507.06186$]$

[76] Yu.A. Shibanov, G.G. Pavlov, V.E. Zavlin, J. Ventura, Model atmospheres and radiation of magnetic neutron stars. I. The fully ionized case, Astron. Astrophys. 266 (1992) 313

[77] V.E. Zavlin, G.G. Pavlov, Yu.A. Shibanov, J. Ventura, Thermal radiation from rotating neutron star: effect of the magnetic field and surface temperature distribution, Astron. Astrophys. 297 (1995) 441

[78] Yu.N. Gnedin \& R.A. Sunyaev, Polarization of optical and X-radiation from compact thermal sources with magnetic field, Astron. Astrophys. 36 (1974) 379

[79] S. Zane, R. Turolla, L. Stella, A. Treves, Proton cyclotron features in thermal spectra of ul- 
tramagnetized neutron stars, Astrophys. J. 560 (2001) 384 [arXiv: astro-ph / 0103316 ]

[80] M. van Adelsberg \& D. Lai, Atmosphere models of magnetized neutron stars: QED effects, radiation spectra and polarization signals, Mon. Not. R. Astron. Soc. 373 (2006) 1495 [arXiv:astro-ph/0607168]

[81] D. Lai \& W.C.G. Ho, Resonant conversion of photon modes due to vacuum polarization in a magnetized plasma: implications for X-ray emission from magnetars, Astrophys. J. 566 (2002) 373 [arXiv:astro-ph/0108127]

[82] W.C.G. Ho, A.Y. Potekhin, G. Chabrier, Model X-ray spectra of magnetic neutron stars with hydrogen atmospheres, Astrophys. J. Suppl. Ser. 178 (2008) 102 [arXiv: 0802 .2957]

[83] M. Rajagopal, R. Romani, M.C. Miller, Magnetized iron atmospheres for neutron stars, Astrophys. J. 479 (1997) 347 [arXiv: astro-ph/9610177]

[84] W.C.G. Ho, NSMAXG: A new magnetic neutron star spectral model in XSPEC, in proceedings of the IAU Symposium 302 Magnetic Fields Throughout Stellar Evolution, ed. by M. Jardine, P. Petit, H.C. Spruit. Cambridge University Press, Cambridge (2014) 435 [arXiv:1311.5583]

[85] J.P. Halpern \& E.V. Gotthelf, Spin-down measurement of PSR J1852+0040 in Kesteven 79: central compact objects as anti-magnetars, Astrophys. J. 709 (2010) 436

[86] E.V. Gotthelf, J.P. Halpern, J. Alford, The spin-down of PSR J0821-4300 and PSR J12105226: Confirmation of central compact objects as anti-magnetars, Astrophys. J. 765 (2013) 58 [arXiv: 1301.2717]

[87] W.C.G. Ho, Central compact objects and their magnetic fields, in proceedings of the IAU Symposium 291 Neutron Stars and Pulsars: Challenges and Opportunities after 80 years, ed. by J. van Leeuven. Cambridge University Press, Cambridge (2013) 101 [arXiv: 1210.7112]

[88] M.A. Ruderman, Matter in superstrong magnetic fields: the surface of a neutron star, Phys. Rev. Lett. 27 (1971) 1306

[89] Z. Medin \& D. Lai, Density-functional-theory calculations of matter in strong magnetic fields. II. Infinite chains and condensed matter, Phys. Rev. A 74 (2006) 062508 [arXiv:astro-ph/0607277]

[90] W. Ebeling \& G. Norman, Coulombic phase transitions in dense plasmas, J. Stat. Phys. 110 (2003) 861 [arXiv: cond-mat/0210193]

[91] E.E. Salpeter, Energy and pressure of a zero-temperature plasma, Astrophys. J. 134 (1961) 669

[92] Z. Medin \& D. Lai, Condensed surfaces of magnetic neutron stars, thermal surface emission, and particle acceleration above pulsar polar caps, Mon. Not. R. Astron. Soc. 382 (2007) 1833 [arXiv:0708.38]

[93] N. Itoh, Electrical conductivity and emissivity of the pulsar surface, Mon. Not. R. Astron. Soc. 173 (1975) $1 \mathrm{P}$ 
[94] R. Lenzen \& J. Trümper, Reflection of X rays by neutron star surfaces, Nature 271 (1978) 216

[95] W. Brinkmann, Thermal radiation from highly magnetized neutron stars, Astron. Astrophys. 82 (1980) 352

[96] R. Turolla, S. Zane, J.J. Drake, Bare quark stars or naked neutron stars? The case of RX J1856.5-3754, Astrophys. J. 603 (2004) 265 [arXiv:astro-ph/0308326]

[97] J.F. Pérez-Azorín, J.A. Miralles, J.A. Pons, Thermal radiation from magnetic neutron star surfaces, Mon. Not. R. Astron. Soc. 433 (2005) 275 [arXiv: astro-ph / 0410664 ]

[98] M. van Adelsberg, D. Lai, A.Y. Potekhin, P. Arras, Radiation from condensed surface of magnetic neutron stars, Astrophys. J. 628 (2005) 902 [arXiv: astro-ph/ 0406001 ]

[99] A.Y. Potekhin, V.F. Suleimanov, M. van Adelsberg, K. Werner, Radiative properties of magnetic neutron stars with metallic surfaces and thin atmospheres, Astron. Astrophys. 546 (2012) A121 [arXiv:1208.6582]

[100] C. Motch, V.E. Zavlin, F. Haberl, The proper motion and energy distribution of the isolated neutron star RX J0720.4-3125, Astron. Astrophys. 408 (2003) 323 [arXiv:astro-ph/0305016]

[101] W.C.G. Ho, D.L. Kaplan, P. Chang, M. van Adelsberg, A.Y. Potekhin, Magnetic hydrogen atmosphere models and the neutron star RX J1856.5-3754, Mon. Not. R. Astron. Soc. 375 (2007) 821 [arXiv:astro-ph/0612145]

[102] V. Suleimanov, A.Y. Potekhin, K. Werner, Models of magnetized neutron star atmospheres: thin atmospheres and partially ionized hydrogen atmospheres with vacuum polarization, Astron. Astrophys. 500 (2009) 891 [arXiv: 0905 . 3276]

[103] V. Suleimanov, V. Hambaryan, A.Y. Potekhin, M. van Adelsberg, R. Neuhäuser, K. Werner, Radiative properties of highly magnetized isolated neutron star surfaces and approximate treatment of absorption features in their spectra, Astron. Astrophys. 522 (2010) A111 [arXiv:1006.3292]

[104] G.G. Pavlov \& Yu.A. Shibanov, Thermal emission of an optically thick plasma containing a strong magnetic field, Sov. Astron. 22 (1978) 43

[105] G.G. Pavlov \& V.E. Zavlin, Polarization of thermal X-rays from isolated neutron stars, Astrophys. J. 529 (2000) 1011 [arXiv:astro-ph/9909326]

[106] D. Lai \& W.C.G. Ho, Polarized X-ray emission from magnetized neutron stars: signature of strong-field vacuum polarization, Phys. Rev. Lett. 91 (2003) 071101 [arXiv:astro-ph/0303596]

[107] M. van Adelsberg \& R. Perna, Soft X-ray polarization in thermal magnetar emission, Mon. Not. R. Astron. Soc. 399 (2009) 1523 [arXiv: 0907.3499 ]

[108] R. Taverna, F. Muleri, R. Turolla, P. Soffitta, S. Fabiani, L. Nobili, Probing magnetar magnetosphere through X-ray polarization measurements, Mon. Not. R. Astron. Soc. 438 (2014) 1686 [arXiv:1311.7500]

[109] S. Chandrasekhar, Radiative Transfer. Clarendon Press, Oxford (1950) 
[110] D. Lai \& W.C.G. Ho, Transfer of polarized radiation in strongly magnetized plasmas and thermal emission from magnetars: effect of vacuum polarization, Astrophys. J. 588 (2003) 962 [arXiv:astro-ph/0211315]

[111] J.S. Heyl \& N. Shaviv, Polarization evolution in strong magnetic fields, Mon. Not. R. Astron. Soc. 311 (2000) 555 [arXiv:astro-ph/9909339]

[112] J.S. Heyl \& N. Shaviv, QED and the high polarization of the thermal radiation from neutron stars, Phys. Rev. D 66 (2002) 023002 [arXiv: astro-ph/9909339]

[113] J.S. Heyl, N. Shaviv, D. Lloyd, The high-energy polarization-limiting radius of neutron star magnetospheres - I. Slowly rotating neutron stars, Mon. Not. R. Astron. Soc. 342 (2003) 134 [arXiv:astro-ph/0302118]

[114] R. Taverna, R. Turolla, D. Gonzalez Caniulef, S. Zane, F. Muleri, P. Soffitta, Polarization of neutron star surface emission: a systematic analysis, Mon. Not. R. Astron. Soc. 454 (2015) 3254 [arXiv: 1509.05023 ]

[115] R. Turolla, Isolated neutron stars: the challenge of simplicity, in Neutron Stars and Pulsars, ed. by W. Becker. Springer, Berlin (2009) 141

[116] M.M. Hohle, F. Haberl, J. Vink, C.P. de Vries, R. Neuhäuser, Narrow absorption features in the co-added XMM-Newton RGS spectra of isolated neutron stars, Mon. Not. R. Astron. Soc. 419 (2012) 1525 [arXiv:1109.2506]

[117] F.M. Walter, T. Eisenbeiß, J.M. Lattimer, B. Kim, V. Hambaryan, R. Neuhäuser, Revisiting the parallax of the isolated neutron star RX J185635-3754 using HST/ACS imaging, Astrophys. J. 724 (2010) 669 [arXiv: 1008 .1709]

[118] C.O. Heinke, H.N. Cohn, P.M. Lugger, et al., Improved mass and radius constraints for quiescent neutron stars in $\omega$ Cen and NGC 6397, Mon. Not. R. Astron. Soc. 444 (2014) 443 [arXiv:1406.1497]

[119] M.H. van Kerkwijk \& D.L. Kaplan, Timing the nearby isolated neutron star RX J1856.5-3754, Astrophys. J. 673 (2008) L163 [arXiv : 0712 . 3212]

[120] A.J. Deutsch, The electromagnetic field of an idealized star in rigid rotation in vacuo, Ann. d'Astrophys. 18 (1955) 1

[121] A.Y. Potekhin, A.F. Fantina, N. Chamel, J.M. Pearson, S. Goriely, Analytical representations of unified equations of state for neutron-star matter, Astron. Astrophys. 560 (2013) A48 [arXiv: 1310.0049$]$

[122] A. Spitkovsky, Time-dependent force-free pulsar magnetospheres: axisymmetric and oblique rotators, Astrophys. J. 648 (2006) 51 [arXiv : astro-ph/ 0603147 ]

[123] Ü. Ertan, Ş. Çalişkan, O. Benli, M.A. Alpar, Long-term evolution of dim isolated neutron stars, Mon. Not. R. Astron. Soc. 444 (2014) 1559 [arXiv: 1408 . 0650]

[124] W.C.G. Ho, Constraining the geometry of the neutron star RX J1856.5-3754, Mon. Not. R. Astron. Soc. 380 (2007) 71 [arXiv: 0705.4543 ]

[125] V.I. Kondratiev, M.A. McLaughlin, D.R. Lorimer, et al., New limits on radio emission from 
X-ray dim isolated neutron stars, Astrophys. J. 702 (2009) 692 [arXiv: 0907.0054 ]

[126] V. Hambaryan, V. Suleimanov, A.D. Schwope, R. Neuhäuser, K. Werner, A.Y. Potekhin, Phase-resolved spectroscopic study of the isolated neutron star RBS 1223 (1RXS J130848.6+212708), Astron. Astrophys. 534 (2011) A74 [arXiv:1108.3897]

[127] D. Sanwal, G.G. Pavlov, V.E. Zavlin, M.A. Teter, Discovery of absorption features in the X-ray spectrum of an isolated neutron star, Astrophys. J. 574 (2002) L61 [arXiv:astro-ph/0206195]

[128] G.F. Bignami, P.A. Caraveo, A. De Luca, S. Mereghetti, The magnetic field of an isolated neutron star from X-ray cyclotron absorption lines, Nature 423 (2003) 725 [arXiv:astro-ph/0306189]

[129] K. Mori, J.C. Chonko, C.J. Hailey, Detailed spectral analysis of the 260 ks XMM-Newton data of 1E 1207.4-5209 and significance of a $2.1 \mathrm{keV}$ absorption feature, Astrophys. J. 631 (2005) 1082 [arXiv:astro-ph/0407369]

[130] J.P. Halpern \& E.V. Gotthelf, Proper motion and timing of two unusual pulsars: Calvera and 1E 1207.4-5209, Astrophys. J. 812 (2015) 61 [arXiv: 1510.00683 ]

[131] A.M. Pires, C. Motch, R. Turolla, S.B. Popov, A.D. Schwope, A. Treves, New XMM-Newton observation of the thermally emitting isolated neutron star 2XMM J104608.7-594306, Astron. Astrophys. $\mathbf{5 8 3}$ (2015) A117 [arXiv: 1508 .05246]

[132] A. Karpova, D. Zyuzin, A. Danilenko, Yu. Shibanov, Constraining the parameters of the pulsar wind nebula DA 495 and its pulsar with Chandra and XMM-Newton, Mon. Not. R. Astron. Soc. 453 (2015) 2241 [arXiv: 1508 . 00002]

[133] C.-Y. Ng, V.M. Kaspi, W.C.G. Ho, et al., Deep X-ray observations of the young highmagnetic-field radio pulsar J1119-6127 and supernova remnant G292.2-0.5, Astrophys. J. 761 (2012) 65 [arXiv: 1211.2761$]$

[134] N.I. Storch, W.C.G. Ho, D. Lai, S. Bogdanov, C.O. Heinke, The light curve and internal magnetic field of the mode-switching pulsar PSR B0943+10, Astrophys. J. 789 (2014) L27 [arXiv: 1405.1475$]$

[135] A. Kirichenko, A. Danilenko, Yu. Shibanov, P. Shternin, S. Zharikov, D. Zyuzin, Deep optical observations of the $\gamma$-ray pulsar J0357+3205, Astron. Astrophys. 564 (2014) A81 [arXiv: 1402.2246]

[136] A. Danilenko, P. Shternin, A. Karpova, D. Zyuzin, Yu. Shibanov, The $\gamma$-ray pulsar J0633+0632 in X-rays, Publ. Astron. Soc. Australia 32 (2015) e038 [arXiv: 1509.06956 ] 